\title{
An adaptive supervised wide-area backup protection scheme for transmission lines protection
}

\author{
B. Mallikarjuna', P. V. Vardhan Varma' ${ }^{1}$ S. D. Samir ${ }^{1}$, M. Jaya Bharata Reddy ${ }^{1 *}$ and D. K. Mohanta ${ }^{2}$
}

\begin{abstract}
Maloperation of conventional relays is becoming prevalent due to ever increase in complexity of conventional power grids. They are dominant during power system contingencies like power swing, load encroachment, voltage instability, unbalanced loading, etc. In these situations, adaptive supervised wide-area backup protection (ASWABP) plays a major role in enhancing the power system reliability. A balance between security and dependability of protection is essential to maintain the reliability. This paper proposes multi-phasor measurement units (MPMU) based ASWABP scheme that can function effectively during faults besides power system contingencies. MPMU is an extended version of Phasor Measurement Unit (PMU). It is an intelligent electronic device which estimates the synchronized predominant harmonic phasors $(100 \mathrm{~Hz}$ and $150 \mathrm{~Hz})$ along with the fundamental phasors $(50 \mathrm{~Hz})$ of three phase voltages and currents with high precision. The proposed ASWABP scheme can detect the fault, identify the parent bus, determine the faulty branch and classify the faults using MPMU measurements at System Protection Center (SPC). Based on these MPMU measurements (received at phasor data concentrator (PDC) at SPC) the appropriate relays will be supervised to enhance the overall reliability of the power grid. Numerous case studies are conducted on WSCC-9 bus and IEEE-14 bus systems to illustrate the security and dependability attributes of proposed ASWABP scheme in MATLAB/Simulink environment. Also, comparative studies are performed with the existing conventional distance protection (Mho relays) for corroborating the superiority of the proposed scheme regarding security and dependability. Comparative studies have shown that the proposed scheme can be used as adaptive supervised wide-area backup protection of conventional distance protection.
\end{abstract}

Keywords: Adaptive supervised wide-area backup protection (ASWABP), Multi-phasor measurement unit (MPMU), Security, Dependability

\section{Introduction}

The ever-increasing demand for electricity causes conventional power grids to operate at their maximum operating limit. When the system is under such stressed condition, the occurrence of a static phenomenon like load encroachment or dynamic phenomena like power swings may result in maloperation of distance relays [1-5]. This paves the way for fairly reduced security in power system protection. The abridged security increases the dependability of the protection system [6]. However, balanced measures of security and dependability in a protection system are required

\footnotetext{
*Correspondence: jayabharat_res@yahoo.co.in

'Department of Electrical and Electronics Engineering, National Institute of

Technology, Tiruchirappalli, Tamilnadu, India

Full list of author information is available at the end of the article
}

for the reliable operation of the power system under any operating conditions [7]. Therefore, developing an adaptive coordinated control of the protection system is a growing need for maintaining security-dependability balance $[8,9]$. Emerging synchrophasor measurements based supervised backup protection methodologies can effectively maintain this balance and hence enhances reliability of the power system $[10,11]$.

Inordinate research attention is being received towards synchrophasor measurements based backup protection methods for transmission lines. Major research works rely on phasor measurement unit (PMU) based wide area measurement system (WAMS) and wide area protection system (WAPS) [12-17]. WAMS and WAPS incorporated in backup protection systems were named 
as a wide-area backup protection (WABP) system [18-23]. P.K. Nayak et.al, in their research work [18] have developed a WABP system based on sequence components of the bus voltages. With this proposed system, the authors could identify the bus nearer to the fault and faulty line. Based on these data, the relays to be operated are recognized. Hinge et.al [19] have developed WABP, which works based on segregated phase comparison technique. However, the methodologies proposed in $[18,19]$ may require recalibration of existing relays in terms of pickup criterion sensitivity. Hall et.al [20] have proposed a wide-area distributed current differential scheme for WABP of the transmission line. However, the accuracy of the proposed scheme is affected by the power system unbalances. Though, the differential protection is simple in principle, but not so easy for implementation because it depends on data synchronization on both sides of the line to be protected. $\mathrm{Li}$ et.al in their research work [21] have evaluated the performance of WABP under various power system disturbances. The research works proposed in [20, 21] requires PMUs to be placed at all the buses. Sheng et.al in [22], have implemented numerical relays based on intelligent electronic devices in coordination with other relay agents for maintaining security-dependability balance. J. Ma et.al [23] have developed a fitting factor based WABP. A fault condition is identified based on the higher value of the ratio between protection fitness function and protection fitness expectation function. To avoid maloperation of the relays with multi-zone protection during power swing condition, swing blocking and operating components must be added in Intelligent Electronic Devices (IEDs) [22, 23].

The proposed research methodology attempts to overcome the limitations and shortcomings of methodologies discussed above. The ASWABP scheme can effectively detect and classify the faults using harmonic phasorestimates $(50 \mathrm{~Hz}, 100 \mathrm{~Hz}$ and $150 \mathrm{~Hz})$ of three phase current signals obtained from MPMU for the backup protection of transmission lines. Equivalent harmonic coefficients (EHC), $\mathrm{I}_{\mathrm{EHC}}$ of branches at all buses is calculated to identify the parent bus in the power system. Once the parent bus is identified, the deviation of EHC of all branches w.r.t parent bus is calculated to determine the faulty branch and corresponding relays to be supervised. Furthermore, the transient events other than faults such as power swings and load encroachment along with unbalanced load are detected by using the magnitude of the fundamental current phasor besides the second and third order harmonic current phasors magnitude. The performance of the proposed scheme is validated for different fault conditions (fault resistance (FR), fault inception angle (FIA) \& fault distance (FD)) and other than fault events by carrying out extensive simulations on WSCC-9 bus and IEEE-14 bus systems.

\section{Multi-phasor measurement unit}

MPMU is an intelligent electronic device which can estimate the fundamental and predominant harmonic phasors $\left(2^{\text {nd }}\right.$ and $3^{\text {rd }}$ order) of three phase voltage and current signals. During a fault condition, waveform distortion, as well as the system unbalance occurs. These disturbances results the line voltages and currents to be non-sinusoidal in wave shape, consisting of second and third order harmonics as predominant [24-27]. Selection of $2^{\text {nd }}$ and $3^{\text {rd }}$ harmonic current phasors for fault analysis palys a major role in segregating the fault and non-faulted conditions. The major functional elements of MPMU are shown in Fig. 1, which is used for extracting the fundamental and harmonic current phasors. From Fig. 1 the analog three phase voltages and currents are converted into appropriate voltages by instrument transformers (CVT and CT) [6]. These signals are passed through the anti-aliasing filter to avoid the aliasing phenomenon. Analog to digital converter (ADC) converts these analog signals into digital signals. The phaselocked oscillator (PLO) ensures the time stamping of the voltage and current signals with the help of Global Positioning System (GPS) pulse per second. Microprocessor based multi-phasor estimator calculates the fundamental, second and third order harmonic phasors of three phase voltage and current signals using Discrete Fourier Transform (DFT).

Since fundamental, second and third order harmonic phasors of current signals are deployed in this paper for ASWABP, estimations of these current phasors are

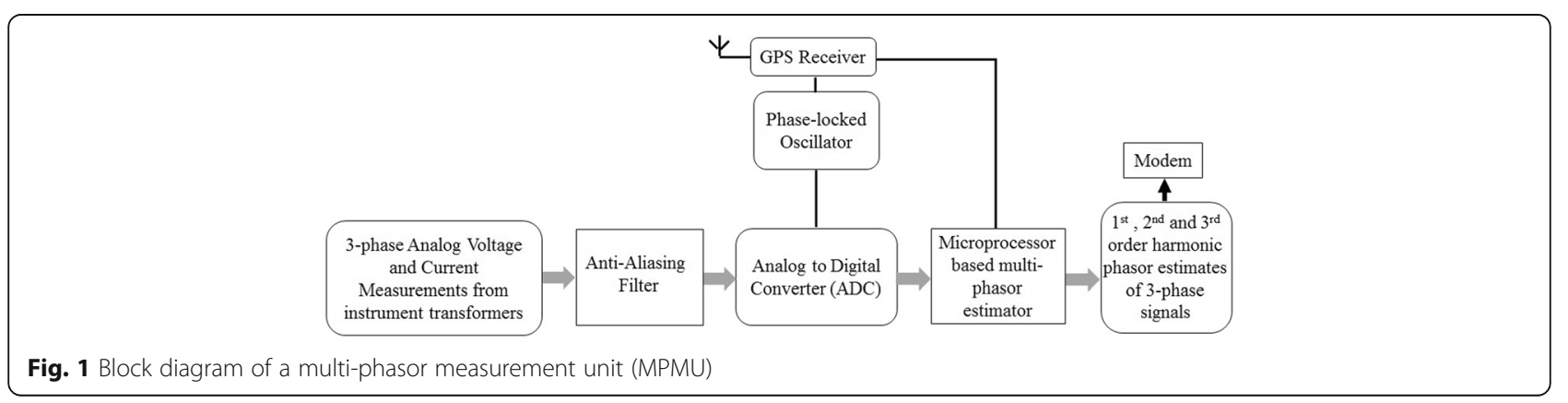


discussed below. DFT of discretized data obtained from $\mathrm{ADC}(i(n \Delta T))$ is given by Eq. (1).

$$
I(k)=\frac{\sqrt{2}}{N} \sum_{n=0}^{N-1} i(n \Delta T) e^{-j \frac{2 \pi k n}{N}} \text { for } \mathrm{k}=0,1, . . \mathrm{N}-1
$$

where,

$\mathrm{N}=$ Number of samples per cycle,

$\mathrm{n}=$ sample number,

$\Delta \mathrm{T}=$ sampling time $=(1 /$ sampling frequency $)$

$=\left(1 / \mathrm{f}_{\mathrm{s}}\right)=\left(1 /\left(\mathrm{N}^{*} \mathrm{f}_{0}\right)\right)$,

$\mathrm{f}_{0}=$ fundamental frequency,

$\mathrm{k}$ = frequency order in terms of fundamental frequency,

DFT is calculated for every $\mathrm{N}$ number of samples ranging from $\mathrm{n}=0$ to $\mathrm{N}-1$.

The harmonic current phasors are estimated by changing the value of $\mathrm{k}$. For fundamental phasor extraction, the value of $\mathrm{k}$ is unity as given in Eq. (2). For second and third order harmonic phasor extraction, $\mathrm{k}$ holds the value of two and three- respectively as given in Eqs. (3) and (4).

$$
\begin{aligned}
& I(1)=I_{1}=\frac{\sqrt{2}}{N} \sum_{n=0}^{N-1} i(n \Delta T) e^{-j \frac{2 \pi n}{N}} \\
& I(2)=I_{2}=\frac{\sqrt{2}}{N} \sum_{n=0}^{N-1} i(n \Delta T) e^{-j \frac{j \pi n}{N}} \\
& I(3)=I_{3}=\frac{\sqrt{2}}{N} \sum_{n=0}^{N-1} i(n \Delta T) e^{-j \frac{6 \pi n}{N}}
\end{aligned}
$$

The MPMU estimated current phasors at different buses in a Power system network are transmitted to
PDC where SPC takes an appropriate action using ASWABP scheme. The following section discusses the development of proposed ASWABP scheme.

\section{Methods}

\subsection{Adaptive supervised wide-area backup protection} scheme

The backup protection plays a major role in increasing the dependability of the power system protection. This increased dependability is acceptable when the power system is in normal operating condition. However, when the system is being operated at its maximum operating limit, a false trip would result in greater damage. Hence, it is desirable to increase the security attribute of the protection system. The security-dependability balance is essential for reliable operation of a power system [6].

The dependability of the protection system is enhanced if all types of line faults are detected and classified accurately. Once the parent bus is detected then faulty branch will be identified and appropriate relays are supervised. The security is improved if all other events such as power swing, load encroachment and unbalanced loading are detected. The dependability and security attributes of the proposed methodology are elaborated through different case studies conducted on Western System of Co-ordinate Council-9 (WSCC-9) bus system in the subsequent sections. Third-order generator model with IEEE Type-1 exciter and distributed transmission line parameters as mentioned in reference [28] are used for modeling the WSCC- 9 bus system shown in Fig. 2.

The positive and zero sequence parameters for $100 \mathrm{~km}$ length of the transmission line in WSCC-9 bus

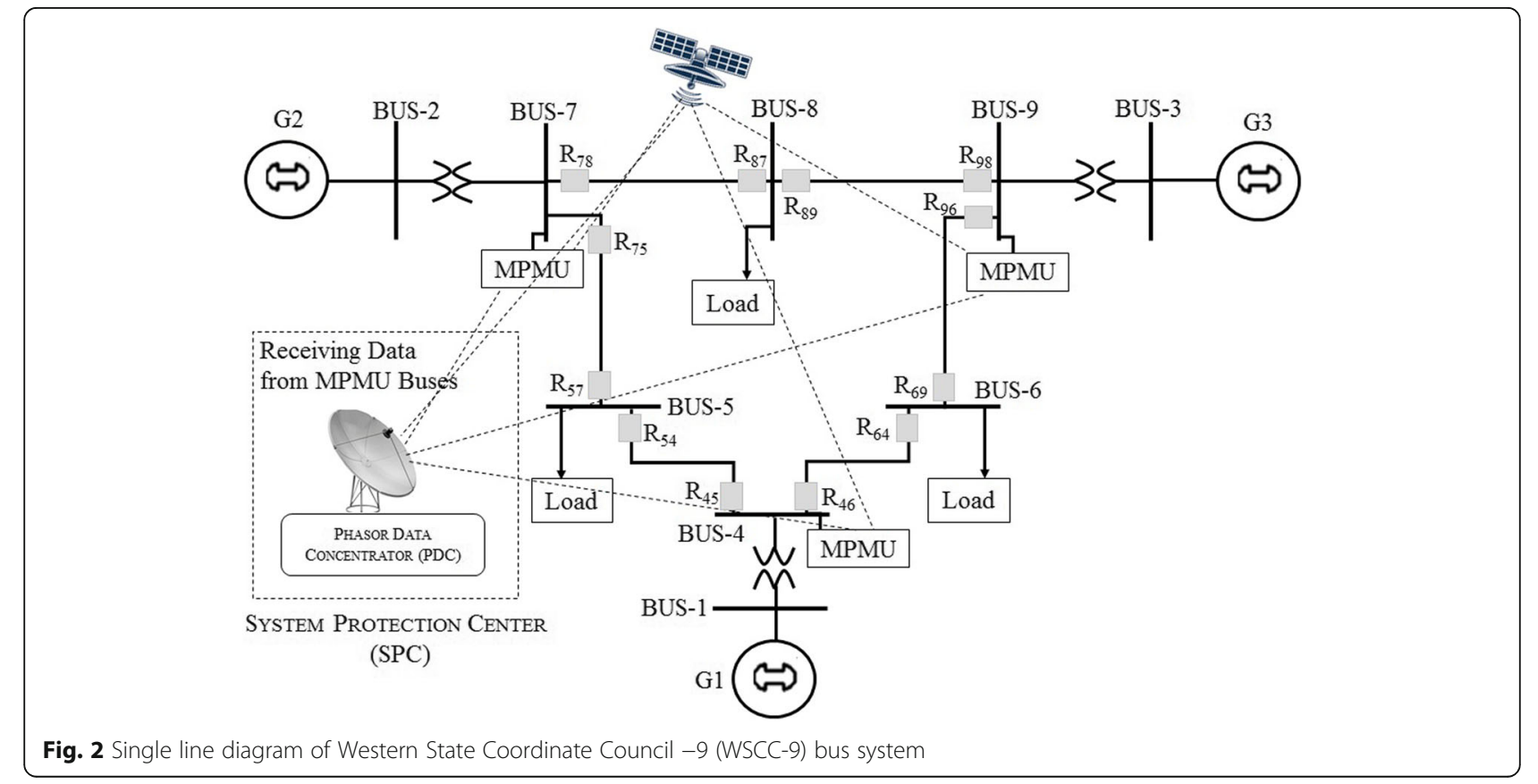


system considered are $\mathrm{R}_{1}=2.34 \Omega, \mathrm{R}_{0}=38.85 \Omega, \mathrm{L}_{1}=$ $95.10 \mathrm{mH}, \quad \mathrm{L}_{0}=325.08 \mu \mathrm{mH}, \mathrm{C}_{1}=1.24 \mu \mathrm{F}, \mathrm{C}_{0}=$ $0.845 \mu \mathrm{F}$ [24]. The values of negative sequence parameters are same as positive sequence parameters. The simulations are carried out in per unit (p.u) system with the base $\mathrm{kV}$ and MVA as $400 \mathrm{kV}$ and 100MVA respectively. MPMUs are installed at BUS-4, BUS-9 and BUS-7 using the optimal PMU location technique proposed for WABP in [29].

\subsubsection{Dependability attribute of proposed methodology}

Dependability attribute of the protection system is a measure of certainty that the protection system would function accordingly for which it is designed to operate [6].

The dependability feature of the proposed backup protection system for transmission line protection is discussed as follows.

As shown in Fig. 3, the estimated multi-phasor current components are sent to PDC in SPC. Fault detection,

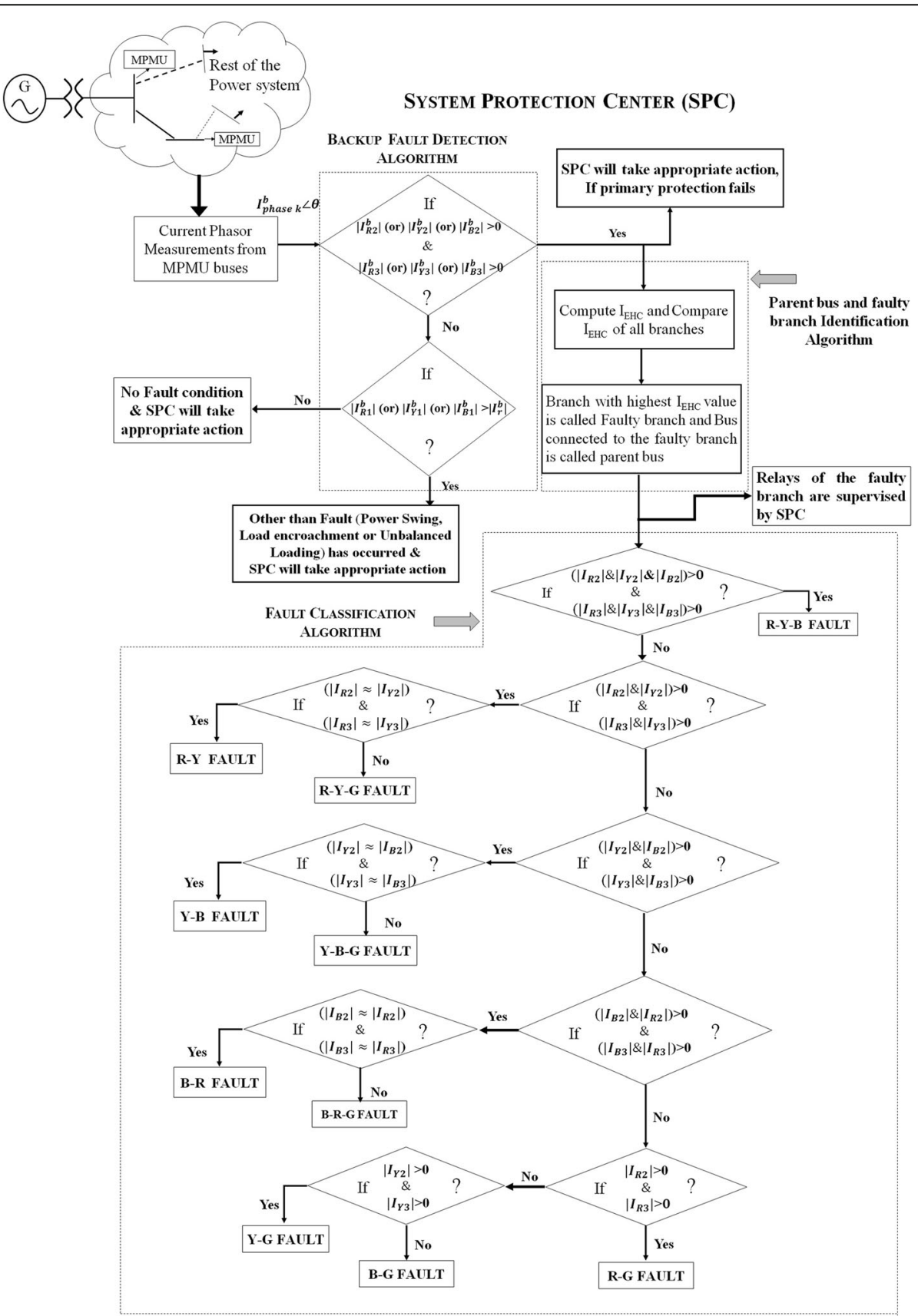

Fig. 3 Sequence of execution of proposed ASWABP Scheme at SPC 
parent bus identification, fault classification, determination of the faulty branch and its relays to be supervised are obtained using ASWABP scheme in SPC. The functional logic of proposed ASWABP scheme for achieving these operations are also illustrated in Fig. 3. The threephase current signals (, , Y \& B) are passed through MPMU to estimate the fundamental $\left(\mathrm{I}_{R 1}^{b}, \mathrm{I}_{Y 1}^{b} \& \mathrm{I}_{B 1}^{b}\right)$, second $\left(\mathrm{I}_{R 2}^{b}, \mathrm{I}_{Y 2}^{b} \& \mathrm{I}_{B 2}^{b}\right)$ and third $\left(\mathrm{I}_{R 3}^{b}, \mathrm{I}_{Y 3}^{b} \& \mathrm{I}_{B 3}^{b}\right)$ order frequency current phasors of a branch ' $\mathrm{b}$ ' connected to MPMU placed bus. The $2^{\text {nd }}\left(\left|I_{R 2}^{b}\right|,\left|I_{Y 2}^{b}\right| \&\left|I_{B 2}^{b}\right|\right)$ and $3^{\text {rd }}(\mid$ $\left.I_{R 3}^{b}|,| I_{Y 3}^{b}|\&| I_{B 3}^{b} \mid\right)$ order harmonic phasors of three-phase current signals are employed to detect the state of the system. During normal condition and stressed conditions the magnitudes of the second and third order current harmonic phasors are zero. However, during fault conditions, the magnitudes of second and third order current harmonic phasors are non zero.

Once the fault is detected, the next stage is identification of parent bus and faulty branch. The bus connected to the faulty branch where MPMU located is termed as the parent bus. The faulty branch will have the highest value of $\mathrm{I}_{\mathrm{EHC}}$ among all branches. Hence, the faulty branch is detected and relays connected to the faulty branch to be supervised are identified. This enhances the dependability attribute of power system protection. The EHC of a particular branch in the system is calculated using Eq. (5),

$$
I_{E H C}=\sqrt{\sum_{k=2,3}\left(I_{R k}^{b}\right)^{2}+\sum_{k=2,3}\left(I_{Y k}^{b}\right)^{2}+\sum_{k=2,3}\left(I_{B k}^{b}\right)^{2}}
$$

where $I_{R k}^{b}$ is the $\mathrm{k}^{\text {th }}$ order harmonic phasor in R-phase current of a branch 'b'. Similarly, $I_{Y k}^{b}$ and $I_{B k}^{b}$ represent the $\mathrm{k}^{\text {th }}$ order harmonic phasors in $\mathrm{Y}$ and $\mathrm{B}$ phase currents of the branch bus ' $b$ ' respectively.

Once the parent bus is identified and the faulty branch is detected then fault classification algorithm is executed as illustrated in Fig. 3. If the magnitudes of all $2^{\text {nd }}\left(I_{R 2}\right.$, $\mathrm{I}_{\mathrm{Y} 2}$, and $\left.\mathrm{I}_{\mathrm{B} 2}\right)$ and $3^{\text {rd }}\left(\mathrm{I}_{\mathrm{R} 3}, \mathrm{I}_{\mathrm{Y} 3}\right.$ and $\left.\mathrm{I}_{\mathrm{B} 3}\right)$ order harmonic phasors of three phase currents $(\mathrm{R}, \mathrm{Y}$ and $\mathrm{B})$ are greater than zero, identified fault is RYB. Else, if the magnitudes of predominant harmonic phasors of $\mathrm{R}$ and $\mathrm{Y}$ phase currents are greater than zero, identified fault is either RY or RYG. The type of fault is further distinguished in the following way: if $\left|I_{R 2}\right|$ is equal to $\left|I_{Y 2}\right|$, and $\left|I_{R 3}\right|$ is equal to $\left|I_{Y 3}\right|$, the classified fault is RY. Otherwise, it is RYG fault. The detected fault is either YB or YBG if the magnitudes of a second ( $\mathrm{I}_{\mathrm{Y} 2}$ and $\mathrm{I}_{\mathrm{B} 2}$ ) and third order harmonic phasors $\left(\mathrm{I}_{\mathrm{Y} 3}\right.$ and $\mathrm{I}_{\mathrm{B} 3}$ ) of $\mathrm{Y}$ and $\mathrm{B}$ phase currents are greater than zero. Further, it is classified as YB if $\left|\mathrm{I}_{\mathrm{Y} 2}\right| \approx\left|\mathrm{I}_{\mathrm{B} 2}\right|$, and $\left|\mathrm{I}_{\mathrm{Y} 3}\right| \approx\left|\mathrm{I}_{\mathrm{B} 3}\right|$. Otherwise, it is YBG fault. The identified fault is either BR or BRG if $\left(\left|I_{B 2}\right| \&\left|I_{R 2}\right|\right)$ and $\left(\left|I_{B 3}\right| \&\left|I_{R 3}\right|\right)$ are greater than zero. Furthermore, it is classified as a $B R$ fault, if $\left|I_{B 2}\right| \approx\left|I_{R 2}\right|$, and $\left|I_{B 3}\right| \approx\left|I_{R 3}\right|$. Otherwise, it is identified as a BRG fault. Detected fault will be RG if the magnitudes of predominant harmonic phasors $\left(I_{R 2}\right.$ and $\left.I_{R 3}\right)$ of $R$-phase current alone are greater than zero. Else the fault is YG if $2^{\text {nd }}\left(\left|\mathrm{I}_{Y 2}\right|\right)$ and $3^{\text {rd }}\left(\left|\mathrm{I}_{\mathrm{Y} 3}\right|\right)$ order phasors of $\mathrm{Y}$-phase current are higher than zero. Otherwise, the fault is BG.

Case studies are carried out with different faults with various fault conditions for validating the dependability attribute of the proposed ASWABP scheme in the WSCC- 9 bus system. Results of the case studies are given in Table 1. For instance, from Table 1, when a line to line (L-L) fault in branch 9-8 with FR of $0 \Omega$ and FIA of $0^{0}$ at $50 \mathrm{~km}$ from BUS- 9 occurs, the magnitude of $2^{\text {nd }}$ and $3^{\text {rd }}$ order harmonic current phasor estimates of almost all branches of MPMU buses are higher than zero. Hence, a fault is detected. After fault detection, EHC of all branches at MPMU buses is calculated and tabulated. From these results, it is observed that branch $(9-8)$ has higher $\mathrm{I}_{\mathrm{EHC}}$ value as compared with that of all other branches. Hence, the branch (9-8) is the faulty branch. Branch $9-8$ is connected between BUS-9 and BUS- 8 since MPMU is connected to BUS-9, it is considered as the parent bus. The relays of the faulty branch $9-8, R_{98}$ $\& R_{89}$ are supervised by SPC. Simultaneously, the fault classification algorithm is executed on $\left|I_{R 2}\right|,\left|I_{Y 2}\right|,\left|I_{B 2}\right|$, $\left|I_{R 3}\right|,\left|I_{Y 3}\right|$ and $\left|I_{B 3}\right|$ of branch 9-8. These harmonic current phasors hold the values as 1.081, 1.08, 0, 0.77, 0.78 and 0 respectively. The detected fault is either RY or RYG since $\left(\left|I_{R 2}\right| \&\left|I_{Y 2}\right|\right)$ and $\left(\left|I_{R 3}\right| \&\left|I_{Y 3}\right|\right)$ are higher than zero. Further, the fault is distinguished as RY because of $\left|\mathrm{I}_{\mathrm{R} 2}\right| \approx\left|\mathrm{I}_{\mathrm{Y} 2}\right|$ and $\left|\mathrm{I}_{\mathrm{R} 3}\right| \approx\left|\mathrm{I}_{\mathrm{Y} 3}\right|$. Likewise, the proposed backup fault detection, parent bus identification, relays of the faulty branch to be supervised along with classification algorithms are performed well for all LG, LLL, LLG with different fault conditions as portrayed in Table 1. Also, the performance of ASWABP scheme under no fault condition is observed and tabulated in Table 1 . It is observed from the table that under No fault condition, the magnitudes of second and third order harmonic phasors currents are zero.

From the above case studies, it is observed that the proposed methodology is instrumental in enhancing the dependability of the protection system.

\subsubsection{Security attribute of proposed methodology}

When transmission system is under stressed condition (like power swing, load encroachment and unbalanced loading, etc.), the insecure operation of protection system would result in greater damage to the system. In such conditions, it is desirable to alter the bias of the protection system in favor of increased security with a slightly increased possibility that the primary protection would not work as designed in case of a fault. So, 
Table 1 Results of case studies, various faults with different fault conditions, conducted on WSCC-9 bus system

\begin{tabular}{|c|c|c|c|c|c|c|c|c|c|c|}
\hline \multirow[t]{2}{*}{ Fault condition } & \multirow{2}{*}{$\begin{array}{l}\text { Bus } \\
\text { number } \\
\text { where } \\
\text { MPMU } \\
\text { is } \\
\text { installed }\end{array}$} & \multirow{2}{*}{$\begin{array}{l}\text { Connected } \\
\text { branches } \\
\text { with } \\
\text { MPMU } \\
\text { installed } \\
\text { bus }\end{array}$} & \multicolumn{3}{|c|}{$\begin{array}{l}\text { Measurements of phasor currents from } \\
\text { MPMU connected branch }\end{array}$} & \multirow{2}{*}{$\begin{array}{l}\text { Is fault detected? } \\
\left(\left(\left|\left\|_{R 2}|\&|\right\|_{R 31}\right) \text { or }\right.\right. \\
\left(||_{Y_{2}}|\&| \|_{Y 3} \mid\right) \text { or } \\
\left(\left\|_{B 2}|\&|\right\|_{B 3} \mid\right)>0 \\
(Y / N)\end{array}$} & \multirow[t]{2}{*}{||$_{E H C} \mid$} & \multirow{2}{*}{$\begin{array}{l}\text { Parent bus, } \\
\text { Faulty } \\
\text { branch } \\
\text { between } \\
\text { two buses }\end{array}$} & \multirow[t]{2}{*}{$\begin{array}{l}\text { Relays to be } \\
\text { supervised }\end{array}$} & \multirow{2}{*}{$\begin{array}{l}\text { Fault } \\
\text { type } \\
\text { classifiec }\end{array}$} \\
\hline & & & ||$_{R 1}|,|\left\|_{R 2}|,|\right\|_{R 3} \mid$ & $\left.\left.|| Y_{1}||\right|_{,Y_{2}}||\right|_{,Y_{3}} \mid$ & ||$_{B 1}|,| \|\left._{B 2}||\right|_{B 3} \mid$, & & & & & \\
\hline \multirow{6}{*}{$\begin{array}{l}\text { L-G fault in branch 4-5 } \\
\text { with } F R=100 \Omega \text {, } \\
\text { and FIA }=72^{\circ} \text { at } 80 \\
\text { km from BUS-4 }\end{array}$} & \multirow[t]{2}{*}{4} & $4-5$ & $3.23,0.89,0.41$ & $1.23,0,0$ & $1.26,0,0$ & \multirow[t]{6}{*}{ Y } & 0.993 & \multirow{6}{*}{$\begin{array}{l}\text { BUS-4, } \\
\text { Branch 4-5 }\end{array}$} & \multirow{6}{*}{$R_{45} \& R_{54}$} & \multirow[t]{6}{*}{$\mathrm{RG}$} \\
\hline & & $4-6$ & $3.56,0.09,0.063$ & $3.98,0,0$ & $4.03,0,0$ & & 0.108 & & & \\
\hline & \multirow[t]{2}{*}{7} & $7-5$ & $4.09,0.423,0.34$ & $2.64,0,0$ & $2.68,0,0$ & & 0.54 & & & \\
\hline & & $7-8$ & $1.71,0.114,0.09$ & $2.15,0,0$ & $2.18,0,0$ & & 0.145 & & & \\
\hline & \multirow[t]{2}{*}{9} & $9-8$ & $0.26,0.05,0.04$ & $0.24,0,0$ & $0.19,0,0$ & & 0.064 & & & \\
\hline & & $9-6$ & $1.99,0.216,0.19$ & $1.72,0,0$ & $1.74,0,0$ & & 0.285 & & & \\
\hline \multirow{6}{*}{$\begin{array}{l}\text { L-L fault in branch 9-8 } \\
\text { with } F R=0 \Omega \text { and FIA }=0^{0} \\
\text { at } 50 \mathrm{~km} \text { from BUS-9 }\end{array}$} & \multirow[t]{2}{*}{4} & $4-5$ & $0.73,0.093,0.07$ & $1.79,0.09,0.07$ & $1.22,0,0$ & \multirow[t]{6}{*}{ Y } & 0.161 & \multirow{6}{*}{$\begin{array}{l}\text { BUS-9, } \\
\text { Branch 9-8 }\end{array}$} & \multirow{6}{*}{$R_{98} \& R_{89}$} & \multirow[t]{6}{*}{ RY } \\
\hline & & $4-6$ & $2.97,0.096,0.08$ & $2.07,0.1,0.08$ & $2.55,0,0$ & & 0.178 & & & \\
\hline & \multirow[t]{2}{*}{7} & $7-5$ & $5.0,0.289,0.217$ & $3.27,0.29,0.22$ & $2.172,0,0$ & & 0.511 & & & \\
\hline & & $7-8$ & $10.77,0.77,0.19$ & $12.2,0.87,0.29$ & $3.96,0,0$ & & 1.209 & & & \\
\hline & \multirow[t]{2}{*}{9} & $9-8$ & $13.8,1.081,0.77$ & $13.9,1.08,0.78$ & $0.244,0,0$ & & 1.88 & & & \\
\hline & & $9-6$ & $2.41,0.266,0.18$ & $3.89,0.27,0.18$ & $1.67,0,0$ & & 0.455 & & & \\
\hline \multirow{6}{*}{$\begin{array}{l}\text { L-L-G fault in branch 4-6 } \\
\text { with } F R=45 \Omega \text { and } \\
\text { FIA }=162^{\circ} \text { at } 50 \mathrm{~km} \\
\text { from BUS-4 }\end{array}$} & \multirow[t]{2}{*}{4} & $4-5$ & $1.73,0.276,0.1$ & $1.61,0.24,0.05$ & $1.28,0,0$ & \multirow[t]{6}{*}{ Y } & 0.382 & BUS-4, & $R_{46} \& R_{64}$ & RYG \\
\hline & & $4-6$ & $4.57,1.399,0.39$ & $4.4,1.074,0.18$ & $1.281,0,0$ & & 1.845 & & & \\
\hline & 7 & $7-5$ & $1.01,0.36,0.024$ & $1.86,0.32,0.01$ & $2.55,0,0$ & & 0.484 & & & \\
\hline & & $7-8$ & $0.87,0.345,0.12$ & $0.78,0.3,0.08$ & $0.278,0,0$ & & 0.479 & & & \\
\hline & 9 & $9-8$ & $4.24,0.36,0.101$ & $4.34,0.29,0.02$ & $1.891,0,0$ & & 0.476 & & & \\
\hline & & $9-6$ & $4.12,1.044,0.34$ & $4.3,0.99,0.12$ & $2.349,0,0$ & & 1.489 & & & \\
\hline L-L-L fault in branch 7-8 & 4 & $4-5$ & $2.06,0.49,0.08$ & $2.09,0.28,0.02$ & $2.01,0.55,0.06$ & Y & 0.789 & BUS-7, & $R_{78} \& R_{87}$ & RYB \\
\hline $\begin{array}{l}\text { With } F R=60 \Omega 2 \text { and } \\
F \mid A=54^{\circ} \text { at BUS-8 }\end{array}$ & & $4-6$ & $3.94,0.198,0.05$ & $3.95,0.11,0.02$ & $3.91,0.19,0.03$ & & 0.304 & Branch $7-8$ & & \\
\hline & 7 & $7-5$ & $1.34,0.55,0.167$ & $1.46,0.4,0.05$ & $1.26,0.67,0.22$ & & 0.994 & & & \\
\hline & & $7-8$ & $5.84,1.89,0.361$ & $5.77,1.18,0.17$ & $1.26,2.19,0.37$ & & 3.176 & & & \\
\hline & 9 & $9-8$ & $2.39,0.938,0.16$ & $2.45,0.58,0.03$ & $2.34,1.09,0.14$ & & 1.571 & & & \\
\hline & & $9-6$ & $0.26,0.18,0.052$ & $0.25,0.15,0.01$ & $0.25,0.24,0.06$ & & 0.344 & & & \\
\hline No Fault Condition & 4 & $4-5$ & $1.091,0,0$ & $1.091,0,0$ & $1.091,0,0$ & $\mathrm{~N}$ & --- & --- & --- & --- \\
\hline & & $4-6$ & $2.129,0,0$ & $2.129,0,0$ & $2.129,0,0$ & & & & & \\
\hline & 7 & $7-5$ & $2.719,0,0$ & $2.719,0,0$ & $2.719,0,0$ & & & & & \\
\hline & & $7-8$ & $1.722,0,0$ & $1.722,0,0$ & $1.722,0,0$ & & & & & \\
\hline & 9 & $9-8$ & $2.226,0,0$ & $2.226,0,0$ & $2.226,0,0$ & & & & & \\
\hline & & $9-6$ & $0.264,0,0$ & $0.203,0,0$ & $0.251,0,0$ & & & & & \\
\hline
\end{tabular}

security is defined as the measure of certainty that the protection system would not malfunction [6].

In this paper, the proposed scheme is devised to detect such events and take appropriate decisions under the supervision of SPC to enhance the security of the power system. Such events are detected using the fundamental phasor $\left(\left|\mathrm{I}_{R 1}^{b}\right|,\left|\mathrm{I}_{Y 1}^{b}\right|\right.$ and $\left.\left|\mathrm{I}_{B 1}^{b}\right|\right)$ of three phase currents along with the second and third order harmonic phasors $\left(\left|\mathrm{I}_{R 2}^{b}\right|,\left|\mathrm{I}_{Y 2}^{b}\right|,\left|\mathrm{I}_{B 2}^{b}\right|,\left|\mathrm{I}_{R 3}^{b}\right|,\left|\mathrm{I}_{Y 3}^{b}\right|\right.$ and $\left.\left|\mathrm{I}_{B 3}^{b}\right|\right)$ of connected branch ' $\mathrm{b}$ ' as shown in Fig. 3. If any conditions like power swing, load encroachment, and unbalanced loading occurs, the magnitude of $2^{\text {nd }}$ and $3^{\text {rd }}$ order harmonic current phasors are equal to zero but the magnitude of any one of the fundamental phasors of three phase currents will be more than the rated value. If this condition is detected SPC takes the necessary action. Thereby, the security of the protection system will be enhanced.

For illustrating the security attribute of the proposed scheme power swing, load encroachment and unbalanced loading scenarios are simulated on the WSCC-9 bus system. Case studies on power swing, load 
Table 2 Results of case studies, power system contingencies without and with fault conditions, conducted on WSCC-9 bus system

\begin{tabular}{|c|c|c|c|c|c|c|c|c|c|c|c|}
\hline \multirow[t]{2}{*}{$\begin{array}{l}\text { Power System } \\
\text { Condition }\end{array}$} & \multirow{2}{*}{$\begin{array}{l}\text { Bus } \\
\text { number } \\
\text { where } \\
\text { MPMU } \\
\text { is } \\
\text { installed }\end{array}$} & \multirow{2}{*}{$\begin{array}{l}\text { Connected } \\
\text { branches } \\
\text { with } \\
\text { MPMU } \\
\text { installed } \\
\text { bus }\end{array}$} & \multicolumn{3}{|c|}{$\begin{array}{l}\text { Measurements of phasor } \\
\text { currents from MPMU } \\
\text { connected branch }\end{array}$} & \multirow{2}{*}{$\begin{array}{l}\text { Is fault detected? } \\
\left(\left(||_{R 2}|\&| \|_{R 3}\right) \text { or }\right. \\
\left(\left.||_{Y 2}|\&|\right|_{Y 3}\right) \text { or } \\
\left(||_{B 2} \&||_{B 3} \mid\right)>0 \\
(Y / N)\end{array}$} & \multirow{2}{*}{||$_{\mathrm{EHC}} \mid$} & \multirow{2}{*}{$\begin{array}{l}\text { Is other } \\
\text { than fault } \\
\text { detected? } \\
\left(\left|\|_{R_{1} \mid}\right| \text { or }\left|\|_{Y_{1}}\right|\right. \\
\left.\text { or }\left|\|_{B_{1}}\right|\right)> \\
|| r \mid(Y / N)\end{array}$} & \multirow{2}{*}{$\begin{array}{l}\text { Parent bus, } \\
\text { Faulty } \\
\text { branch } \\
\text { between } \\
\text { two buses }\end{array}$} & \multirow[t]{2}{*}{$\begin{array}{l}\text { Relays } \\
\text { to be } \\
\text { supervised }\end{array}$} & \multirow[t]{2}{*}{$\begin{array}{l}\text { Fault } \\
\text { type } \\
\text { Classified }\end{array}$} \\
\hline & & & $\begin{array}{l}\left|\left\|_{R 1}|,|\right\|_{R 2}\right|_{1} \\
\left|\|_{R 3}\right|\end{array}$ & $\begin{array}{l}\|\left.\left.\right|_{Y_{1}}\right|_{1} \\
\mid\left\|_{Y_{2}}\right\|_{,} \\
\left.||_{Y_{3}}\right|^{\prime}\end{array}$ & $\begin{array}{l}\left.||_{B 1}|,|\right|_{B 2} \mid, \\
||_{B 3} \mid\end{array}$ & & & & & & \\
\hline \multirow[t]{6}{*}{$\begin{array}{l}\text { Power Swing } \\
\text { without fault }\end{array}$} & \multirow[t]{2}{*}{4} & $4-5$ & $1.614,0,0$ & $\begin{array}{l}1.545 \\
0,0\end{array}$ & $\begin{array}{l}1.512 \\
0,0\end{array}$ & \multirow[t]{6}{*}{ N } & \multirow[t]{6}{*}{--} & \multirow[t]{6}{*}{$Y$} & \multirow[t]{6}{*}{ Branch 9-6 } & \multirow[t]{6}{*}{$R_{96} \& R_{69}$} & \multirow[t]{6}{*}{---} \\
\hline & & $4-6$ & $0,0,0$ & $0,0,0$ & $0,0,0$ & & & & & & \\
\hline & \multirow[t]{2}{*}{7} & $7-5$ & $\begin{array}{l}0.506 \\
0,0\end{array}$ & $\begin{array}{l}0.47 \\
0,0\end{array}$ & $\begin{array}{l}0.331 \\
0,0\end{array}$ & & & & & & \\
\hline & & $7-8$ & $\begin{array}{l}2.044 \\
0,0\end{array}$ & $\begin{array}{l}2.097 \\
0,0\end{array}$ & $\begin{array}{l}2.145 \\
0,0\end{array}$ & & & & & & \\
\hline & \multirow[t]{2}{*}{9} & $9-8$ & $\begin{array}{l}0.482 \\
0,0\end{array}$ & $\begin{array}{l}0.361 \\
0,0\end{array}$ & $\begin{array}{l}0.409 \\
0,0\end{array}$ & & & & & & \\
\hline & & $9-6$ & $\begin{array}{l}3.525 \\
0,0\end{array}$ & $\begin{array}{l}3.395 \\
0,0\end{array}$ & $\begin{array}{l}3.206 \\
0,0\end{array}$ & & & & & & \\
\hline \multirow{6}{*}{$\begin{array}{l}\text { Power Swing with LLL } \\
\text { fault with FR }=100 \Omega \text {, } \\
\text { FIA }=36^{\circ} \text { in branch } 7-5 \\
\text { at } 20 \mathrm{~km} \text { from BUS-7 }\end{array}$} & \multirow[t]{2}{*}{4} & $4-5$ & $\begin{array}{l}2.492 \\
0.312 \\
0.115\end{array}$ & $\begin{array}{l}2.274 \\
0.422 \\
0.147\end{array}$ & $\begin{array}{l}2.204 \\
0.303 \\
0.216\end{array}$ & \multirow[t]{6}{*}{ Y } & 0.669 & \multirow[t]{6}{*}{---} & $\begin{array}{l}\text { BUS-7, } \\
\text { Branch 7-5 }\end{array}$ & \multirow[t]{6}{*}{$R_{75} \& R_{57}$} & \multirow[t]{6}{*}{ RYB } \\
\hline & & $4-6$ & $0,0,0$ & $0,0,0$ & $0,0,0$ & & 0 & & & & \\
\hline & \multirow[t]{2}{*}{7} & $7-5$ & $\begin{array}{l}7.149 \\
0.352 \\
0.411\end{array}$ & $\begin{array}{l}6.171 \\
1.086 \\
0.759\end{array}$ & $\begin{array}{l}5.912, \\
1.296 \\
0.949\end{array}$ & & 2.151 & & & & \\
\hline & & $7-8$ & $\begin{array}{l}1.191 \\
0.38 \\
0.01\end{array}$ & $\begin{array}{l}1.61 \\
0.424 \\
0.144\end{array}$ & $\begin{array}{l}1.555 \\
0.214 \\
0.104\end{array}$ & & 0.634 & & & & \\
\hline & \multirow[t]{2}{*}{9} & $9-8$ & $\begin{array}{l}0.264 \\
0.06, \\
0.019\end{array}$ & $\begin{array}{l}0.203, \\
0.074 \\
0.054\end{array}$ & $\begin{array}{l}0.251 \\
0.075 \\
0.055\end{array}$ & & 0.145 & & & & \\
\hline & & $9-6$ & $\begin{array}{l}2.431 \\
0.138 \\
0.151\end{array}$ & $\begin{array}{l}2.22 \\
0.313 \\
0.112\end{array}$ & $\begin{array}{l}2.02 \\
0.419 \\
0.291\end{array}$ & & 0.642 & & & & \\
\hline \multirow[t]{6}{*}{$\begin{array}{l}\text { Load encroachment } \\
\text { without fault }\end{array}$} & \multirow[t]{2}{*}{4} & $4-5$ & $\begin{array}{l}0.943 \\
0,0\end{array}$ & $\begin{array}{l}0.92 \\
0,0\end{array}$ & $\begin{array}{l}0.969 \\
0,0\end{array}$ & \multirow[t]{6}{*}{$N$} & -- & Y & Branch 7-8 & $R_{78} \& R_{87}$ & --- \\
\hline & & $4-6$ & $\begin{array}{l}0.389 \\
0,0\end{array}$ & $\begin{array}{l}0.713 \\
0,0\end{array}$ & $\begin{array}{l}0.516 \\
0,0\end{array}$ & & & & & & \\
\hline & 7 & $7-5$ & $\begin{array}{l}2.055 \\
0,0\end{array}$ & $\begin{array}{l}1.949 \\
0,0\end{array}$ & $\begin{array}{l}2.029 \\
0,0\end{array}$ & & & & & & \\
\hline & & $7-8$ & $\begin{array}{l}5.335 \\
0,0\end{array}$ & $\begin{array}{l}5.804 \\
0,0\end{array}$ & $\begin{array}{l}5.346 \\
0,0\end{array}$ & & & & & & \\
\hline & 9 & $9-8$ & $\begin{array}{l}2.85 \\
0,0\end{array}$ & $\begin{array}{l}2.886 \\
0,0\end{array}$ & $\begin{array}{l}2.84 \\
0,0\end{array}$ & & & & & & \\
\hline & & $9-6$ & $\begin{array}{l}1.876 \\
0,0\end{array}$ & $\begin{array}{l}1.926 \\
0,0\end{array}$ & $\begin{array}{l}1.868 \\
0,0\end{array}$ & & & & & & \\
\hline $\begin{array}{l}\text { Load encroachment } \\
\text { with LLL fault with } \\
F R=50 \Omega, F I A=0^{\circ}\end{array}$ & 4 & $4-5$ & $\begin{array}{l}2.769 \\
0.354 \\
0.304\end{array}$ & $\begin{array}{l}2.718 \\
0.112 \\
0.068\end{array}$ & $\begin{array}{l}2.264 \\
0.459 \\
0.346\end{array}$ & Y & 0.752 & --- & $\begin{array}{l}\text { BUS-7, } \\
\text { Branch 7-8 }\end{array}$ & $R_{78} \& R_{87}$ & RYB \\
\hline at $15 \mathrm{~km}$ from BUS-7 & & $4-6$ & $\begin{array}{l}3.724, \\
0.414, \\
0.346\end{array}$ & $\begin{array}{l}3.558 \\
0.16 \\
0.08\end{array}$ & $\begin{array}{l}3.102, \\
0.571 \\
0.409\end{array}$ & & 0.903 & & & & \\
\hline & 7 & $7-5$ & $\begin{array}{l}0.776 \\
0.262 \\
0.231\end{array}$ & $\begin{array}{l}1.222 \\
0.225 \\
0.186\end{array}$ & $\begin{array}{l}1.194 \\
0.482 \\
0.406\end{array}$ & & 0.777 & & & & \\
\hline & & $7-8$ & $\begin{array}{l}9.745 \\
0.949 \\
0.816\end{array}$ & $\begin{array}{l}9.875 \\
0.407 \\
0.322\end{array}$ & $\begin{array}{l}8.391 \\
1.359 \\
1.144\end{array}$ & & 2.234 & & & & \\
\hline
\end{tabular}


Table 2 Results of case studies, power system contingencies without and with fault conditions, conducted on WSCC-9 bus system (Continued)

\begin{tabular}{|c|c|c|c|c|c|c|c|c|c|}
\hline & 9 & $9-8$ & $\begin{array}{l}6.026 \\
0.859 \\
0.17\end{array}$ & $\begin{array}{l}7.153 \\
0.369 \\
0.19\end{array}$ & $\begin{array}{l}6.512 \\
0.793 \\
0.228\end{array}$ & 1.273 & & & \\
\hline & & $9-6$ & $\begin{array}{l}1.365 \\
0.125 \\
0.132\end{array}$ & $\begin{array}{l}2.0 \\
0.089 \\
0.062\end{array}$ & $\begin{array}{l}1.82 \\
0.195 \\
0.159\end{array}$ & 0.329 & & & \\
\hline \multirow[t]{6}{*}{$\begin{array}{l}\text { Unbalanced } \\
\text { loading }\end{array}$} & 4 & $4-5$ & $\begin{array}{l}1.492 \\
0,0\end{array}$ & $\begin{array}{l}1.274 \\
0,0\end{array}$ & $1.2,0,0 \quad \mathrm{~N}$ & --- & $\begin{array}{l}---, \\
\text { Branch 7-8 }\end{array}$ & $R_{78} \& R_{87}$ & -- \\
\hline & & $4-6$ & $\begin{array}{l}2.891 \\
0,0\end{array}$ & $\begin{array}{l}2.844 \\
0,0\end{array}$ & $\begin{array}{l}2.831 \\
0,0\end{array}$ & & & & \\
\hline & 7 & $7-5$ & $\begin{array}{l}1.19 \\
0,0\end{array}$ & $\begin{array}{l}1.61 \\
0,0\end{array}$ & $\begin{array}{l}1.555 \\
0,0\end{array}$ & & & & \\
\hline & & $7-8$ & $\begin{array}{l}4.149 \\
0,0\end{array}$ & $\begin{array}{l}2.171 \\
0,0\end{array}$ & $\begin{array}{l}3.912, \\
0,0\end{array}$ & & & & \\
\hline & 9 & $9-8$ & $\begin{array}{l}2.431 \\
0,0\end{array}$ & $\begin{array}{l}2.22, \\
0,0\end{array}$ & $\begin{array}{l}2.021 \\
0,0\end{array}$ & & & & \\
\hline & & $9-6$ & $\begin{array}{l}0.342 \\
0,0\end{array}$ & $\begin{array}{l}0.342 \\
0,0\end{array}$ & $\begin{array}{l}0.342 \\
0,0\end{array}$ & & & & \\
\hline
\end{tabular}

encroachment with and without faults along with unbalanced loading are given in Table 2 .

For instance, consider power swing condition when branch 4-6 is disconnected from the system due to some abnormal condition. The measurements of all branches connected to all MPMU buses are given in Table 2. From Table 2, the magnitude of fundamental phasors of $3-\Phi$ current signals $\left(\left|\mathrm{I}_{R 1}^{b}\right|,\left|\mathrm{I}_{Y 1}^{b}\right|,\left|\mathrm{I}_{B 1}^{b}\right|\right)$ of all branches of MPMU buses are higher than rated value. However, the magnitudes of the predominant current harmonic phasors $\left(\left|\mathrm{I}_{R 2}^{b}\right|,\left|\mathrm{I}_{Y 2}^{b}\right|,\left|\mathrm{I}_{B 2}^{b}\right|,\left|\mathrm{I}_{R 3}^{b}\right|,\left|\mathrm{I}_{Y 3}^{b}\right|\right.$ and $\left.\left|\mathrm{I}_{B 3}^{b}\right|\right)$ are zero. When these values are passed through the proposed scheme, it is identified as other than fault condition because none of the second and third order harmonic current phasors satisfy the fault detection criterion, and the magnitudes of the fundamental current phasors are more than the rated values. Among all branches of MPMU buses, branch 9-6 of BUS-9 has higher magnitudes of fundamental current phasors, hence the relays $\mathbf{R}_{\mathbf{9 6}}$ and $\mathbf{R}_{\mathbf{6 9}}$ of the branch $6-9$ are instructed (by SPC) not to operate. Similarly, the proposed algorithm can perform well even under load encroachment and unbalanced conditions as given in Table 2. The corresponding results tabulated in Table 2, validate the security attribute of the proposed algorithm. Hence, the proposed algorithm can enhance the security of the protection system.

Further, fault conditions during power swing and load encroachment are also studied, and the presence of harmonics can be observed. Results of these case studies are also tabulated in Table 2. The fault detection, parent bus identification, determination of faulty branch relays to be supervised and fault classification methods remain same as described in the preceding section. Similar analysis can be carried as illustrated in the algorithm, for all other case studies (LG, LL and LLL) and faults are classified even during power system contingencies.

From the above discussions on backup fault detection, parent bus identification, determination of the faulty branch, classification of faults and detection of power system contingencies, it is inferred that the SPC takes an appropriate decision for enhancing the security and dependability attributes of the protection system using wide-area current phasor measurements. In other words, the reliability of the protection system can be enhanced.

\section{Results and discussions}

In general practice, for the traditional backup protection of long transmission lines, conventional distance relays are employed with over-reaching zones [30]. These relay take local measurements for decision making and do not consider fault resistance. Moreover, their characteristics may not be adjustable to changing power system conditions [31, 32]. Hence, they are very prone to maloperate even for high impedance faults and power system contingency conditions. In this section, performance of the proposed scheme and distance protection (Mho relays) under various power system disturbances is studied on the IEEE-14 bus system. Also, for the same case studies, a comparative analysis is carried in terms of security and dependability measures of conventional and proposed protection systems. The transmission line parameters, generator and exciter model parameters of the IEEE-14 bus system are same as WSCC-9 bus 


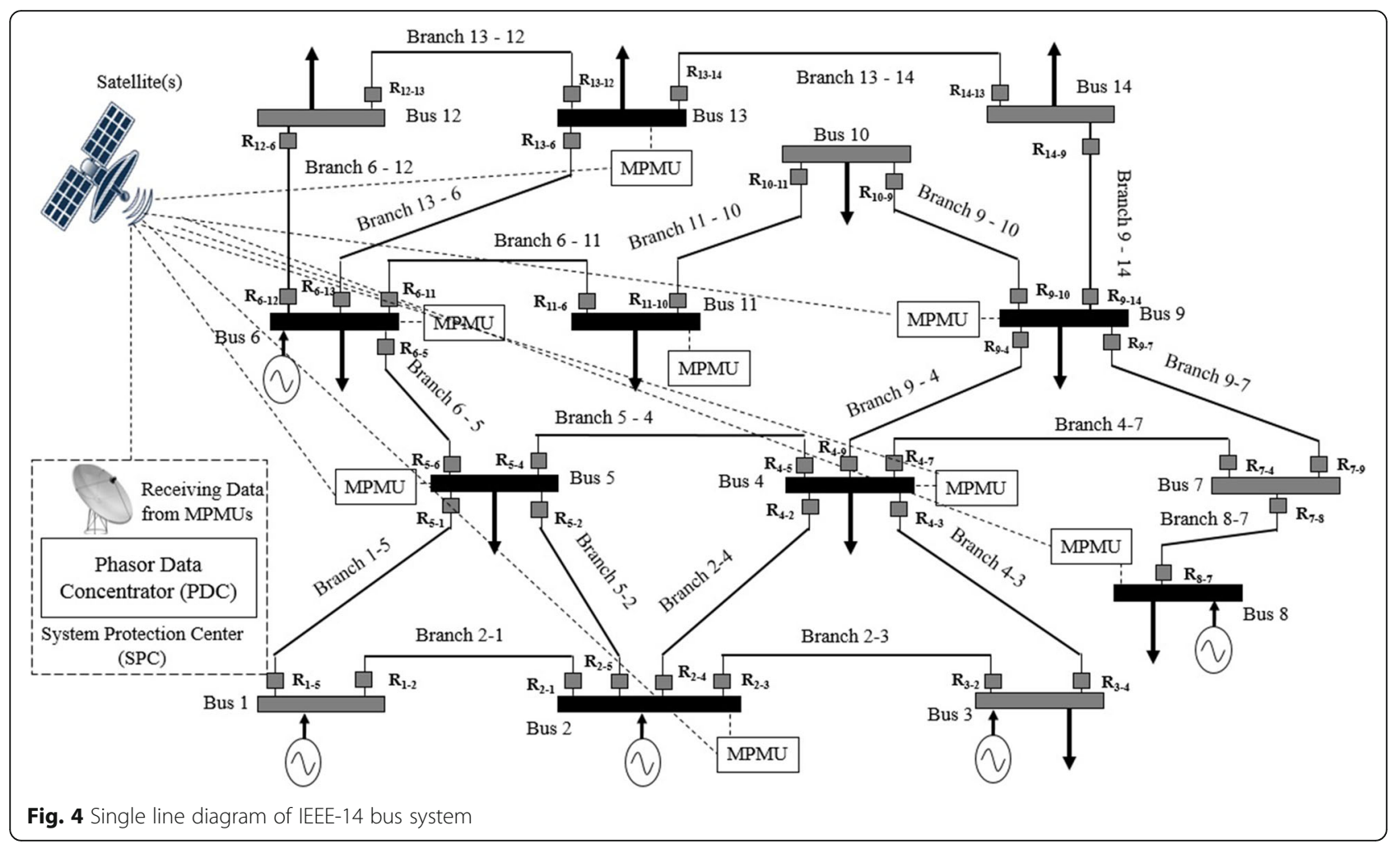

system. MPMUs are strategically located in IEEE-14 bus system at buses 2, 4, 5, 6, 8, 9, 11 and 13 [29]. The single line diagram of the IEEE-14 bus system with MPMUs installed is shown in Fig. 4. Measurements of all branches obtained from MPMU buses are collected at SPC with the help of PDC are analyzed for power system disturbances.

\subsection{The performance of the proposed ASWABP scheme during various power system disturbances}

Numerous case studies on faults and power system contingencies are simulated on the IEEE-14 bus system. In this subsection, a few case studies are presented and analyzed. For better clarity, the branch measurements with significant magnitudes of fundamental, second and third order harmonic phasors of three-phase current signals are tabulated in Tables 3 and 4 .

Table 3 portrays the results of different faults with different fault conditions along with no fault condition. From Table 3, it is observed that all faults are properly detected, the corresponding parent bus is identified, relays of the faulty branch are identified and type of fault is classified. The corresponding measurements are processed through the proposed algorithm as illustrated in section 3. Further, case studies of power system contingencies (power swing and load encroachment with and without faults and unbalanced loading) conducted on IEEE-14 bus system are given in Table 4. It is observed from Table 4 that during these contingencies without fault, the magnitudes of second and third order harmonic current phasors are equal to zero, but that of fundamental current phasors are greater than the rated values. Hence, the proposed scheme has detected them as other than fault (power system contingency) events. The proposed scheme has performed as well when a fault occurs during power system contingency conditions as given in Table 4. In all the power system disturbances, SPC takes appropriate decision and guides the corresponding relays as given Tables 3 and 4 .

\subsection{The performance of the distance protection (Mho relays) scheme during various power system disturbances}

The impedance trajectories of the distance relay for the case studies (for IEEE-14 bus system) as given in Tables 3 and 4, are shown in Figs. 5 and 6. When an LG fault occurred in branch 13-12, (with fault conditions as given in Table 3 ) the relay, $\mathrm{R}_{13-12}$ must observe the impedance in Zone-2 as per its settings, however, due to high fault impedance the fault point has not entered any of the zones as shown in Fig. 5a. Thus, the relay $R_{13-12}$ located at branch $13-12$ is unable to operate as per settings. Similarly, for double 
Table 3 Results of case studies conducted on IEEE-14 bus system under various transmission line faults

\begin{tabular}{|c|c|c|c|c|c|c|c|c|c|c|}
\hline \multirow[t]{2}{*}{ Fault condition } & \multirow{2}{*}{$\begin{array}{l}\text { Bus } \\
\text { number } \\
\text { where } \\
\text { MPMU } \\
\text { is } \\
\text { installed }\end{array}$} & \multirow{2}{*}{$\begin{array}{l}\text { Connected } \\
\text { branches } \\
\text { with } \\
\text { MPMU } \\
\text { installed } \\
\text { bus }\end{array}$} & \multicolumn{3}{|c|}{$\begin{array}{l}\text { Measurements of phasor currents from } \\
\text { MPMU connected branch }\end{array}$} & \multirow{2}{*}{$\begin{array}{l}\text { Is fault detected? } \\
\left(\left(||_{R 2}|\&| \|_{R 3} \mid\right) \text { or }\right. \\
\left(\left.||_{Y 2}|\&|\right|_{Y 3}\right) \text { or } \\
\left(||_{B 2} \&\left|\|_{B 3}\right|\right)>0 \\
(Y / N)\end{array}$} & \multirow[t]{2}{*}{||$_{\text {EHC }} \mid$} & \multirow{2}{*}{$\begin{array}{l}\text { Parent bus, } \\
\text { Faulty } \\
\text { branch } \\
\text { between } \\
\text { two buses }\end{array}$} & \multirow{2}{*}{$\begin{array}{l}\text { Relays to } \\
\text { be } \\
\text { supervised }\end{array}$} & \multirow{2}{*}{$\begin{array}{l}\text { Fault } \\
\text { type } \\
\text { classified }\end{array}$} \\
\hline & & & $\left|\left\|_{R 1}|,|\right\|_{R 2}\right|,\left|\|_{R 3}\right|$ & $\begin{array}{l}\left.\left.||_{Y 1}|,|\right|_{Y 2}\right|_{1} \\
||_{Y 3} \mid\end{array}$ & $\left.\left.||_{B 1}||\right|_{B 2},||\right|_{B 3} \mid$, & & & & & \\
\hline \multirow{8}{*}{$\begin{array}{l}\text { LG fault with FR } \\
\text { of } 70 \Omega \text { \& FIA of } \\
144^{\circ} \text { at } 200 \mathrm{~km} \\
\text { in branch } 13-12 \\
\text { from BUS-13 }\end{array}$} & 2 & $2-5$ & $1.413,0,0$ & $1.66,0,0$ & $1.27,0.52,0.13$ & \multirow[t]{8}{*}{ Y } & 0.558 & \multirow{8}{*}{$\begin{array}{l}\text { BUS-6, } \\
\text { Branch 13- } \\
12\end{array}$} & \multirow{8}{*}{$\begin{array}{l}R_{13-12} \& \\
R_{12-13}\end{array}$} & \multirow[t]{8}{*}{ BG } \\
\hline & 4 & $4-5$ & $1.279,0,0$ & $1.34,0,0$ & $1.12,0.57,0.26$ & & 0.631 & & & \\
\hline & 6 & $6-13$ & $0.48,0,0$ & $0.41,0,0$ & $1.56,0.55,0.16$ & & 0.573 & & & \\
\hline & & $6-12$ & $0.66,0,0$ & $0.136,0,0$ & $1.25,0.67,0.13$ & & 0.74 & & & \\
\hline & 8 & $8-9$ & $0.38,0,0$ & $0.29,0,0$ & $\begin{array}{l}0.34,0.026 \\
0.02\end{array}$ & & 0.043 & & & \\
\hline & 9 & $9-14$ & $0.67,0,0$ & $0.59,0,0$ & $1.98,0.6,0.28$ & & 0.67 & & & \\
\hline & 11 & $11-6$ & $0.66,0,0$ & $0.84,0,0$ & $\begin{array}{l}0.57,0.083 \\
0.19\end{array}$ & & 0.22 & & & \\
\hline & 13 & $13-12$ & $0.15,0,0$ & $2.28,0,0$ & $0.2,1.092,0.36$ & & 1.183 & & & \\
\hline \multirow{8}{*}{$\begin{array}{l}\text { L-L fault with FR } \\
\text { of } 50 \Omega \& \text { FIA of } \\
90^{\circ} \text { at } 0 \mathrm{~km} \text { in } \\
\text { branch } 7-8 \text { from } \\
\text { BUS-7 }\end{array}$} & 2 & $2-4$ & $0.518,0,0$ & $\begin{array}{l}0.42,0.05 \\
0.01\end{array}$ & $0.29,0.07,0.04$ & Y & 0.1 & \multirow[t]{8}{*}{$\begin{array}{l}\text { BUS-8, } \\
\text { Branch 8-7 }\end{array}$} & \multirow[t]{8}{*}{$\begin{array}{l}R_{8-7} \& R_{7-} \\
8\end{array}$} & \multirow[t]{8}{*}{ YB } \\
\hline & 4 & $4-7$ & $2.73,0,0$ & $\begin{array}{l}3.98,1.24 \\
0.12\end{array}$ & $3.18,1.72,0.63$ & & 2.22 & & & \\
\hline & 5 & $5-4$ & $1.41,0,0$ & $\begin{array}{l}1.12,0.561 \\
0.05\end{array}$ & $\begin{array}{l}1.32,0.806 \\
0.19\end{array}$ & & 1.014 & & & \\
\hline & 6 & $6-5$ & $0.73,0,0$ & $\begin{array}{l}0.59,0.112 \\
0.07\end{array}$ & $\begin{array}{l}1.23,0.835 \\
0.14\end{array}$ & & 1.016 & & & \\
\hline & 8 & $8-7$ & $3.57,0,0$ & $\begin{array}{l}2.54,1.64 \\
0.85\end{array}$ & $2.48,1.7,0.88$ & & 2.35 & & & \\
\hline & 9 & $9-7$ & $0.23,0,0$ & $\begin{array}{l}1.19,0.87 \\
0.22\end{array}$ & $1.59,0.97,0.75$ & & 1.52 & & & \\
\hline & 11 & $11-9$ & $0.64,0,0$ & $\begin{array}{l}0.77,0.14 \\
0.01\end{array}$ & $\begin{array}{l}0.63,0.287 \\
0.05\end{array}$ & & 0.327 & & & \\
\hline & 13 & $13-14$ & $0.62,0,0$ & $\begin{array}{l}0.39,0.301 \\
0.09\end{array}$ & $\begin{array}{l}0.472,0.296 \\
0.28\end{array}$ & & 0.517 & & & \\
\hline \multirow{8}{*}{$\begin{array}{l}\text { L-L-G fault with } \\
\text { FR of } 15 \Omega \text { \& FIA } \\
\text { of } 72^{0} \text { at } 45 \mathrm{~km} \\
\text { in branch } 1-2 \\
\text { from BUS-2 }\end{array}$} & 2 & $2-1$ & $0.32,0,0$ & $\begin{array}{l}8.25,3.87 \\
0.98\end{array}$ & $9.65,6.75,2.09$ & Y & 8.11 & \multirow[t]{8}{*}{$\begin{array}{l}\text { BUS-2, } \\
\text { Branch 2-1 }\end{array}$} & \multirow[t]{8}{*}{$\begin{array}{l}R_{2-1} \& R_{1-} \\
2\end{array}$} & \multirow[t]{8}{*}{ YBG } \\
\hline & 4 & $4-5$ & $1.19,0,0$ & $\begin{array}{l}1.08 \\
0.576,0.222\end{array}$ & $\begin{array}{l}2.87,1.041 \\
0.270\end{array}$ & & 1.3 & & & \\
\hline & 5 & $5-1$ & $1.74,0,0$ & $\begin{array}{l}5.27,1.7 \\
0.2\end{array}$ & $4.7,2.8,0.6$ & & 3.3 & & & \\
\hline & 6 & $6-5$ & $0.128,0,0$ & $\begin{array}{l}0.11,0 \\
0.01\end{array}$ & $1.65,0.1,0.2$ & & 0.3 & & & \\
\hline & 8 & $8-7$ & $0.36,0,0$ & $\begin{array}{l}0.33 \\
0.022,0.009\end{array}$ & $\begin{array}{l}0.28,0.066 \\
0.02\end{array}$ & & 0.075 & & & \\
\hline & 9 & $9-4$ & $1.46,0,0$ & $\begin{array}{l}1.42,0.28 \\
0.23\end{array}$ & $\begin{array}{l}1.07,0.543 \\
0.32\end{array}$ & & 0.739 & & & \\
\hline & 11 & $11-6$ & $0.59,0,0$ & $\begin{array}{l}0.55,0.03 \\
0.08\end{array}$ & $0.54,0.07,0.16$ & & 0.21 & & & \\
\hline & 13 & $13-6$ & $0.29,0,0$ & $\begin{array}{l}0.56,0.18 \\
0.04\end{array}$ & $0.32,0.11,0.07$ & & 0.37 & & & \\
\hline \multirow{3}{*}{$\begin{array}{l}\text { L-L-L Fault with } \\
\text { FR of } 60 \Omega \& \text { FIA } \\
\text { of } 0^{\circ} \text { at } 65 \mathrm{~km} \text { in } \\
\text { branch } 9-10 \\
\text { from BUS-9 }\end{array}$} & 2 & $2-5$ & $1.59,0.245,0.191$ & $\begin{array}{l}1.6,0.11 \\
0.049\end{array}$ & $\begin{array}{l}1.6,0.258 \\
0.223\end{array}$ & Y & 0.476 & \multirow[t]{3}{*}{$\begin{array}{l}\text { BUS-9, } \\
\text { Branch 9-1 }\end{array}$} & \multirow[t]{3}{*}{$\begin{array}{l}R_{9-10} \& \\
R_{10-9}\end{array}$} & \multirow[t]{3}{*}{ RYB } \\
\hline & 4 & $4-9$ & $\begin{array}{l}2.87,1.532, \\
0.31\end{array}$ & $\begin{array}{l}2.79,0.842, \\
0.05\end{array}$ & $\begin{array}{l}1.86,0.269 \\
0.34\end{array}$ & & 1.828 & & & \\
\hline & 5 & $5-6$ & $\begin{array}{l}3.04,1.057 \\
0.279\end{array}$ & $\begin{array}{l}3, \\
0.547,0.095\end{array}$ & $3,1.073,0.34$ & & 1.664 & & & \\
\hline
\end{tabular}


Table 3 Results of case studies conducted on IEEE-14 bus system under various transmission line faults (Continued)

\begin{tabular}{|c|c|c|c|c|c|c|c|c|c|}
\hline & 6 & $6-11$ & $\begin{array}{l}3.93,1.532, \\
0.31\end{array}$ & $\begin{array}{l}1.73,0.842 \\
0.05\end{array}$ & $2.971 .551,0.34$ & & 2.382 & & \\
\hline & 8 & $8-7$ & $\begin{array}{l}0.29,0.054 \\
0.03\end{array}$ & $\begin{array}{l}0.32 \\
0.037,0.005\end{array}$ & $0.99,0.054,0.034$ & & 0.097 & & \\
\hline & 9 & $9-10$ & $\begin{array}{l}3.49,1.799 \\
0.199\end{array}$ & $\begin{array}{l}3.37,0.981 \\
0.19\end{array}$ & $3.57,1.807,0.2$ & & 2.746 & & \\
\hline & 11 & $11-10$ & $\begin{array}{l}2.64,1.647, \\
0.155\end{array}$ & $\begin{array}{l}1.53,0.92 \\
0.013\end{array}$ & $\begin{array}{l}2.891 .709 \\
0.148\end{array}$ & & 2.554 & & \\
\hline & 13 & $13-14$ & $\begin{array}{l}2.16,1.065, \\
0.584\end{array}$ & $\begin{array}{l}2.04 \\
0.599,0.017\end{array}$ & $\begin{array}{l}2.19,1.143 \\
0.686\end{array}$ & & 1.901 & & \\
\hline \multirow{8}{*}{$\begin{array}{l}\text { No Fault } \\
\text { Condition }\end{array}$} & 2 & $2-1$ & $0.28,0,0$ & $0.279,0,0$ & $0.272,0,0$ & \multirow[t]{8}{*}{$\mathrm{N}$} & - & \multirow[t]{8}{*}{-} & \multirow[t]{8}{*}{ - } \\
\hline & 4 & 4.5 & $1.15,0,0$ & $1.149,0,0$ & $1.151,0,0$ & & & & \\
\hline & 5 & $5-6$ & $2.01,0,0$ & $2.014,0,0$ & $2.02,0,0$ & & & & \\
\hline & 6 & $6-12$ & $0.57,0,0$ & $0.56,0,0$ & $0.567,0,0$ & & & & \\
\hline & 8 & $8-7$ & $0.35,0,0$ & $0.351,0,0$ & $0.351,0,0$ & & & & \\
\hline & 9 & $9-10$ & $0.129,0,0$ & $0.129,0,0$ & $0.129,0,0$ & & & & \\
\hline & 11 & $11-6$ & $0.585,0,0$ & $0.58,0,0$ & $0.537,0,0$ & & & & \\
\hline & 13 & $13-14$ & $0.196,0,0$ & $0.199,0,0$ & $0.195,0,0$ & & & & \\
\hline
\end{tabular}

line fault (LL) occurred in branch 7-8 with the fault conditions as shown in Table 3, the impedance trajectory of $\mathrm{R}_{7-8}$ is shown in Fig. 5b. From Fig. 5b, the relay $R_{7-8}$ is observing fault point in Zone- 2 instead of Zone-1. Hence, the $R_{7-8}$ is operated with more delay rather than operating instantaneously.

Likewise, for LLG fault occurred in branch 1-2 with fault conditions as given in Table 3 , the relay $R_{2-1}$ is observing the fault in Zone-1, which is the correct operation. For LLL occurred in branch 9-10 with fault conditions as shown in Table 3 , the relay $R_{9-10}$ is observing in Zone-3 instead of Zone-2, because of high impedance fault. The impedance locus of the relays $R_{2-1}$ and $R_{9-10}$ for LLG and LLL faults are shown in Fig. $5 c$ and $5 d$ respectively.

Further, the performance of the distance relays is verified for power swing, load encroachment and unbalanced loading (in IEEE-14 bus system). A Stable power swing is simulated by disconnecting line 10-11. When the line $10-11$ is disconnected, power flowing through line $10-11$ is diverted through the remaining lines connected to BUS-11. Under this condition, the impedance locus enters Zone- 2 of $\mathrm{R}_{6-11}$ as shown in Fig. $6 \mathrm{a}$. Therefore, the relay $R_{6-11}$ will operate as per its time setting though it should not operate.

Load encroachment is created by increasing the load at BUS-3 to $150 \%$. At this condition, the relay $R_{4-3}$ (backup for $\mathrm{R}_{3-2}$ ) has observed the impedance in Zone-3 as shown in Fig. 6b. Furthermore, unbalanced loading is simulated at BUS-12 by loading $\mathrm{R}, \mathrm{Y}$ and $\mathrm{B}$ phases unequally. For this condition, the relays $R_{\mathbf{6 - 1 2}}$ located at BUS- 6 has observed the impedance trajectory in Zone-3 as shown in Fig. 6c.
In the same way, the performance of the conventional distance relays is verified for power swing and load encroachment with faults. The distance relays will not refuse to operate for faults under any one of the above power system contingencies.

From the above studies, it can be inferred that mho relays maloperate for high impedance faults and power system contingencies (power swing, load encroachment and unbalanced loading) without faults. These situations demand adaptive supervised backup protection systems, which are based on wide-area synchrophasor measurements.

\subsection{Comparative analysis of the proposed scheme with distance protection during power system disturbances}

The conventional distance protection algorithms have the following drawbacks, though they are simple and dependable. The disadvantages are reduced security and reliability, fixed adaptability and limited view on power system conditions since their function is based on local measurements. The proposed algorithm can overcome the disadvantages of conventional algorithms as discussed in this section. The only issue associated with the ASWABP scheme operation is latency in data communication from MPMU to PDC (SPC). However, it becomes an inherent advantage for the proposed scheme, since it can be employed as a backup for the existing protection system. A comparative analysis is carried between the proposed scheme and the conventional distance protection relays to quantify the security and dependability measures for the same case studies considered in preceding sections. The inferences of the case studies, simulated for both the schemes are tabulated in Table 5 . 
Table 4 Results of case studies conducted in IEEE-14 bus system under various power system conditions

\begin{tabular}{|c|c|c|c|c|c|c|c|c|c|c|c|c|}
\hline \multirow[t]{2}{*}{$\begin{array}{l}\text { Power System } \\
\text { Condition }\end{array}$} & & \multirow{2}{*}{$\begin{array}{l}\text { Bus } \\
\text { number } \\
\text { where } \\
\text { MPMU } \\
\text { is } \\
\text { installed }\end{array}$} & \multirow{2}{*}{$\begin{array}{l}\text { Connected } \\
\text { branches } \\
\text { with } \\
\text { MPMU } \\
\text { installed } \\
\text { bus }\end{array}$} & \multicolumn{3}{|c|}{$\begin{array}{l}\text { Measurements of phasor } \\
\text { currents from MPMU } \\
\text { connected branch }\end{array}$} & \multirow{2}{*}{$\begin{array}{l}\text { Is fault detected? } \\
\left(\left(\left|\left\|_{R 2} \mid \&\right\|_{R_{3}}\right)\right.\right. \\
\text { or }\left(\left\|\left.\right|_{Y 2}|\&|\right\|_{Y_{3} \mid}\right) \\
\text { or }\left(\left\|_{B 2} \&\left|\|_{B 3}\right|\right)\right) \\
>0 \\
(\mathrm{Y} / \mathrm{N})\end{array}$} & \multirow{2}{*}{$\left|\|_{\text {EHC }}\right|$} & \multirow{2}{*}{$\begin{array}{l}\text { Is other } \\
\text { than fault } \\
\text { detected? } \\
\left(\left|\|_{\mathrm{R} 1}\right| \text { or }\right. \\
\left|\|_{\mathrm{Y} 1}\right| \text { or } \\
\left.\left|\|_{\mathrm{B} 1}\right|\right)>||_{\mathrm{r}} \mid \\
(\mathrm{Y} / \mathrm{N})\end{array}$} & \multirow{2}{*}{$\begin{array}{l}\text { Parent bus, } \\
\text { Faulty } \\
\text { branch } \\
\text { between } \\
\text { two buses }\end{array}$} & \multirow{2}{*}{$\begin{array}{l}\text { Relays } \\
\text { to be } \\
\text { supervised }\end{array}$} & \multirow[t]{2}{*}{$\begin{array}{l}\text { Fault } \\
\text { type } \\
\text { classified }\end{array}$} \\
\hline & & & & 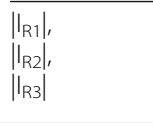 & 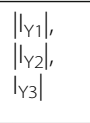 & 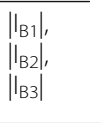 & & & & & & \\
\hline \multirow[t]{16}{*}{$\begin{array}{l}\text { Power } \\
\text { Swing }\end{array}$} & \multirow[t]{8}{*}{$\begin{array}{l}\text { Without } \\
\text { fault }\end{array}$} & 2 & $2-5$ & $0.28,0,0$ & $\begin{array}{l}0.28 \\
0,0\end{array}$ & $\begin{array}{l}0.29,0 \\
0\end{array}$ & \multirow[t]{8}{*}{$\mathrm{N}$} & \multirow[t]{8}{*}{-} & \multirow[t]{8}{*}{ Y } & \multirow{8}{*}{$\begin{array}{l}\text {-, } \\
\text { Branch } \\
6-11\end{array}$} & \multirow[t]{8}{*}{$\begin{array}{l}R_{6-11} \& \\
R_{11-6}\end{array}$} & \multirow[t]{8}{*}{-} \\
\hline & & 4 & $4-5$ & $0.27,0,0$ & $\begin{array}{l}0.28 \\
0,0\end{array}$ & $\begin{array}{l}0.25,0, \\
0\end{array}$ & & & & & & \\
\hline & & \multirow[t]{3}{*}{6} & $6-11$ & $1.7,0,0$ & $\begin{array}{l}1.69 \\
0,0\end{array}$ & $\begin{array}{l}1.68,0 \\
0\end{array}$ & & & & & & \\
\hline & & & $6-13$ & $0.57,0,0$ & $\begin{array}{l}0.57 \\
0,0\end{array}$ & $\begin{array}{l}0.57,0, \\
0\end{array}$ & & & & & & \\
\hline & & & $6-5$ & $0.6,0,0$ & $\begin{array}{l}0.6,0, \\
0\end{array}$ & $0.6,0,0$ & & & & & & \\
\hline & & 8 & $8-7$ & $0.52,0,0$ & $\begin{array}{l}0.51 \\
0,0\end{array}$ & $\begin{array}{l}0.54,0, \\
0\end{array}$ & & & & & & \\
\hline & & \multirow[t]{2}{*}{13} & $13-14$ & $0.58,0,0$ & $\begin{array}{l}0.58 \\
0,0\end{array}$ & $\begin{array}{l}0.58,0, \\
0\end{array}$ & & & & & & \\
\hline & & & $13-12$ & $0.284,0,0$ & $\begin{array}{l}0.284 \\
0,0\end{array}$ & $\begin{array}{l}0.284 \\
0,0\end{array}$ & & & & & & \\
\hline & \multirow{8}{*}{$\begin{array}{l}\text { With fault } \\
\text { (L-L-L } \\
\text { Fault with } \\
\text { FR of } 20 \Omega \\
\& \text { FIA of } \\
0^{0} \text { at } \\
50 \mathrm{~km} \text { in } \\
\text { branch 6- } \\
11 \text { from } \\
\text { BUS-6) }\end{array}$} & 2 & $2-5$ & $\begin{array}{l}0.395,0.09 \\
0.05\end{array}$ & $\begin{array}{l}0.375 \\
0.078 \\
0.017\end{array}$ & $\begin{array}{l}0.233 \\
0.166 \\
0.142\end{array}$ & \multirow[t]{8}{*}{ Y } & 0.263 & \multirow[t]{8}{*}{-} & \multirow[t]{8}{*}{$\begin{array}{l}\text { BUS-6, } \\
\text { Branch 6- } \\
11\end{array}$} & \multirow[t]{8}{*}{$\begin{array}{l}R_{6-11} \& \\
R_{11-6}\end{array}$} & \multirow[t]{8}{*}{ RYB } \\
\hline & & 4 & $4-5$ & $\begin{array}{l}3.829,0.623 \\
0.485\end{array}$ & $\begin{array}{l}3.743 \\
0.056 \\
0.096\end{array}$ & $\begin{array}{l}2.007 \\
0.1226 \\
0.563\end{array}$ & & 0.984 & & & & \\
\hline & & \multirow[t]{2}{*}{6} & $6-5$ & $\begin{array}{l}3.432,0.548, \\
0.44\end{array}$ & $\begin{array}{l}3.264 \\
0.123 \\
0.17\end{array}$ & $\begin{array}{l}2.598 \\
0.66 \\
0.598\end{array}$ & & 1.154 & & & & \\
\hline & & & $6-11$ & $\begin{array}{l}4.82,0.809 \\
0.641\end{array}$ & $\begin{array}{l}4.579 \\
0.187 \\
0.216\end{array}$ & $\begin{array}{l}3.559 \\
0.968 \\
0.824\end{array}$ & & 1.662 & & & & \\
\hline & & 8 & $8-7$ & $\begin{array}{l}0.282,0.012, \\
0.009\end{array}$ & $\begin{array}{l}0.268 \\
0.017 \\
0.027\end{array}$ & $\begin{array}{l}0.267 \\
0.022 \\
0.036\end{array}$ & & 0.055 & & & & \\
\hline & & 9 & $9-10$ & $\begin{array}{l}3.829,0.623 \\
0.485\end{array}$ & $\begin{array}{l}3.743 \\
0.056 \\
0.096\end{array}$ & $\begin{array}{l}2.92 \\
0.66 \\
0.563\end{array}$ & & 1.178 & & & & \\
\hline & & 11 & $11-6$ & $\begin{array}{l}4.755,0.837 \\
0.61\end{array}$ & $\begin{array}{l}4.78 \\
0.148 \\
0.224\end{array}$ & $\begin{array}{l}3.555 \\
0.913 \\
0.617\end{array}$ & & 1.535 & & & & \\
\hline & & 13 & $13-6$ & $\begin{array}{l}4.823,0.741 \\
0.584\end{array}$ & $\begin{array}{l}4.614 \\
0.07 \\
0.039\end{array}$ & $\begin{array}{l}3.713 \\
0.869 \\
0.792\end{array}$ & & 1.51 & & & & \\
\hline \multirow[t]{6}{*}{$\begin{array}{l}\text { Load } \\
\text { Encroachment }\end{array}$} & \multirow[t]{6}{*}{$\begin{array}{l}\text { Without } \\
\text { fault }\end{array}$} & 2 & $2-4$ & $0.28,0,0$ & $\begin{array}{l}0.3,0 \\
0\end{array}$ & $\begin{array}{l}0.28,0, \\
0\end{array}$ & \multirow[t]{6}{*}{$N$} & \multirow[t]{6}{*}{-} & Y & $\overline{-}$, & $\begin{array}{l}R_{4-3} \& R_{3-} \\
4\end{array}$ & - \\
\hline & & 4 & $4-3$ & $2.38,0,0$ & $\begin{array}{l}2.4,0 \\
0\end{array}$ & $\begin{array}{l}2.38,0 \\
0\end{array}$ & & & & & & \\
\hline & & 5 & $5-4$ & $0.646,0,0$ & $\begin{array}{l}0.646 \\
0,0\end{array}$ & $\begin{array}{l}0.643 \\
0,0\end{array}$ & & & & & & \\
\hline & & 6 & $6-5$ & $0.59,0,0$ & $\begin{array}{l}0.58 \\
0,0\end{array}$ & $\begin{array}{l}0.58,0, \\
0\end{array}$ & & & & & & \\
\hline & & 8 & $8-7$ & $0.35,0,0$ & $\begin{array}{l}0.35 \\
0,0\end{array}$ & $\begin{array}{l}0.35,0, \\
0\end{array}$ & & & & & & \\
\hline & & 9 & $9-4$ & $1.43,0,0$ & $\begin{array}{l}1.43 \\
0,0\end{array}$ & $\begin{array}{l}1.44,0 \\
0\end{array}$ & & & & & & \\
\hline
\end{tabular}


Table 4 Results of case studies conducted in IEEE-14 bus system under various power system conditions (Continued)

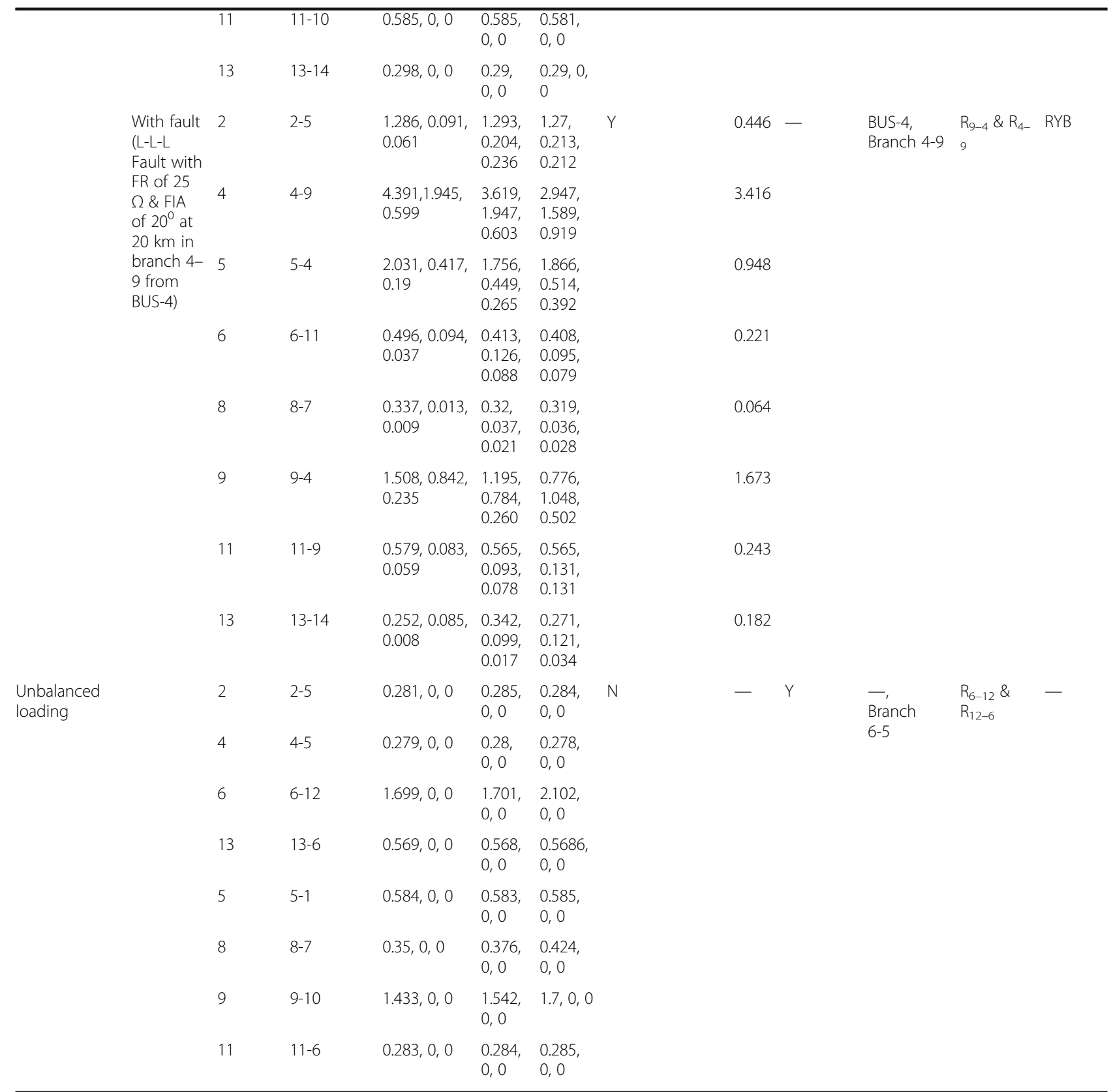

The case studies as depicted in Table 5 illustrate the security and dependability measures of both the schemes. The actions taken by the proposed scheme are desirable and correct for all the case studies whereas that of conventional distance protection are not desirable and incorrect in some cases. For instance, consider LG fault with FR of $70 \Omega$ \& FIA of $144^{\circ}$ at $200 \mathrm{~km}$ in branch 13-12 from BUS-13 (in the IEEE-14 bus system). As illustrated in subsection 4.1 and 4.2, the decision taken by conventional distance protection is not desirable and incorrect. Whereas using proposed ASWABP scheme, the operation of the supervised protective relay will be correct and desirable.

Similarly, for the remaining case studies, it is understood that the performance of the proposed ASWABP is superior over that of the conventional distance protection. Hence, the proposed ASWABP scheme is secure and dependable. The analysis emphasizes the necessity of the proposed scheme as an adaptive supervised widearea backup protection for distance protection. 

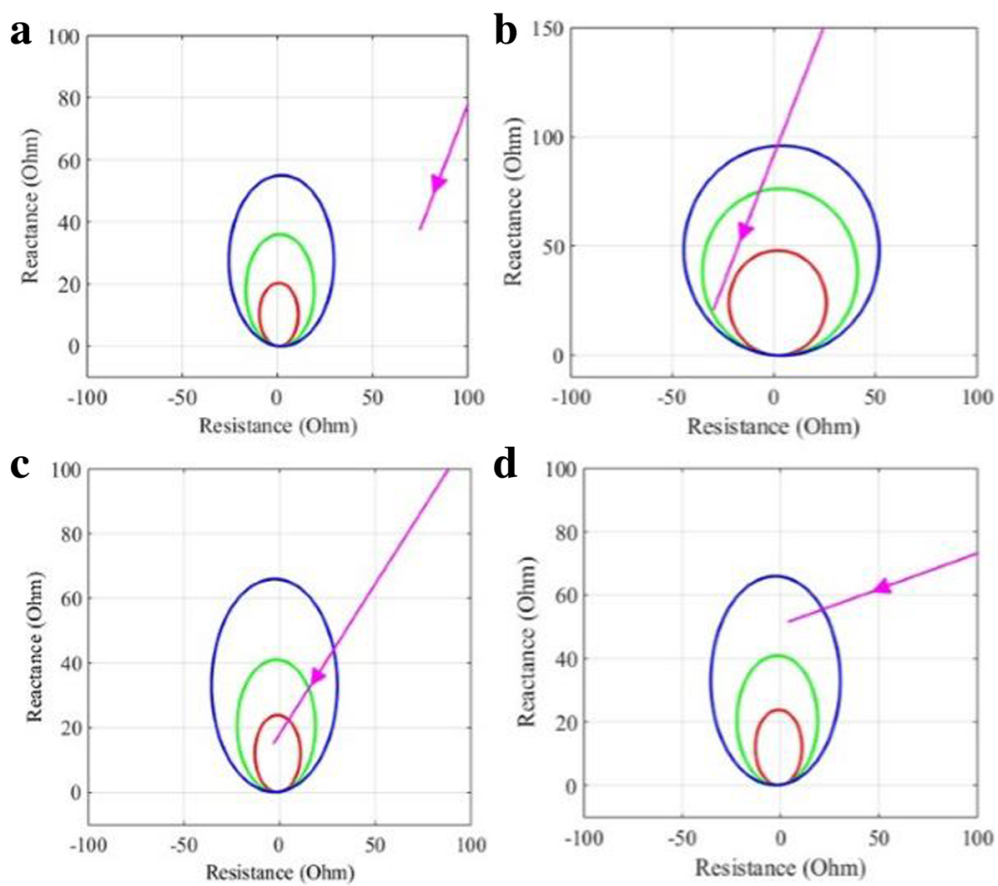

Fig. 5 Impedance trajectory of (a) $R_{13-12}$, when $L G\left(70 \Omega, 144^{\circ}\right.$ and $200 \mathrm{~km}$ from BUS-13) fault occurred; (b) $R_{7-8}$, when $L L\left(50 \Omega, 90^{\circ}\right.$ and $0 \mathrm{~km}$ from

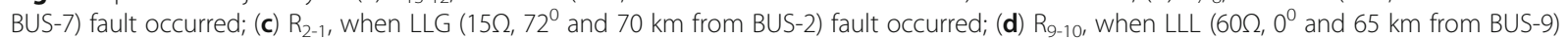
fault occurred
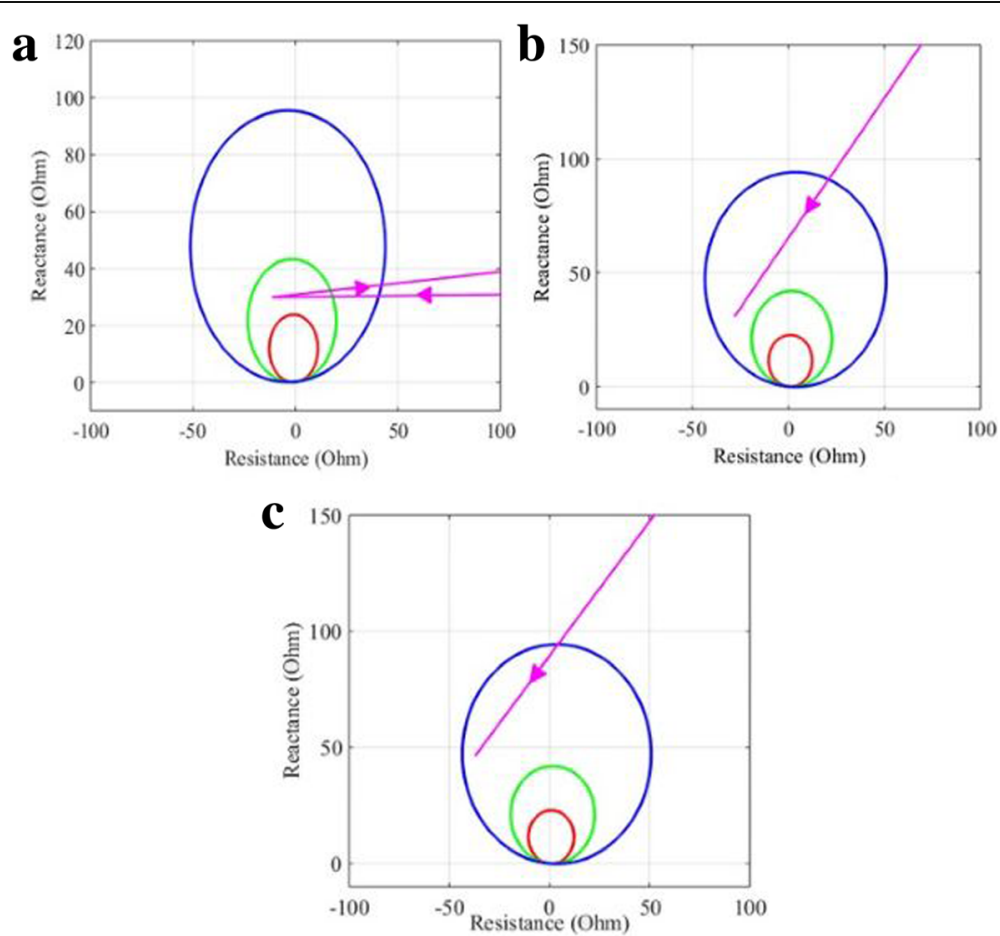

Fig. 6 Impedance trajectory of (a). $R_{6-11}$, when power swing occurred due to loss of line 10-11; (b). $R_{4-3}$, when load encroachment occurred due to overloading at BUS-3; (c). $R_{6-12}$, when unbalanced loading occurred at BUS-12 
Table 5 Comparative analysis of the proposed methodology with conventional distance relay during various power system disturbances in IEEE-14 bus system

\begin{tabular}{|c|c|c|c|c|c|c|}
\hline \multirow[t]{2}{*}{ Power System Disturbances } & \multirow{2}{*}{\multicolumn{2}{|c|}{ Case studies }} & \multicolumn{2}{|c|}{$\begin{array}{l}\text { The action taken by the } \\
\text { proposed scheme is }\end{array}$} & \multicolumn{2}{|c|}{$\begin{array}{l}\text { The action taken by the } \\
\text { conventional distance } \\
\text { protection is }\end{array}$} \\
\hline & & & $\begin{array}{l}\text { Correct } \\
(\mathrm{Y} / \mathrm{N})\end{array}$ & $\begin{array}{l}\text { Desirable } \\
(\mathrm{Y} / \mathrm{N})\end{array}$ & $\begin{array}{l}\text { Correct } \\
(\mathrm{Y} / \mathrm{N})\end{array}$ & $\begin{array}{l}\text { Desirable } \\
(\mathrm{Y} / \mathrm{N})\end{array}$ \\
\hline \multirow[t]{4}{*}{ Fault conditions } & \multicolumn{2}{|c|}{ LG $\left(70 \Omega, 144^{\circ}\right.$ and $200 \mathrm{~km}$ in branch 13-12 from BUS-13) } & Y & Y & $\mathrm{N}$ & N \\
\hline & \multicolumn{2}{|c|}{$\operatorname{LL}\left(50 \Omega, 180^{\circ}\right.$ and $0 \mathrm{~km}$ in branch $4-7$ from BUS-7) } & Y & Y & $\mathrm{N}$ & N \\
\hline & \multicolumn{2}{|c|}{$\operatorname{LLG}\left(15 \Omega, 54^{\circ}\right.$ and $70 \mathrm{~km}$ in branch $1-2$ from BUS-2) } & Y & Y & Y & Y \\
\hline & \multicolumn{2}{|c|}{$\operatorname{LLL}\left(60 \Omega, 0^{\circ}\right.$ and $65 \mathrm{~km}$ in branch 9-10 from BUS-9) } & Y & Y & $\mathrm{N}$ & N \\
\hline \multirow[t]{5}{*}{ Contingency conditions } & \multirow[t]{2}{*}{ Power Swing(at BUS-6) } & without fault & Y & Y & $\mathrm{N}$ & $\mathrm{N}$ \\
\hline & & with fault & Y & Y & Y & Y \\
\hline & \multirow[t]{2}{*}{ Load encroachment (at BUS-3) } & without fault & Y & Y & $\mathrm{N}$ & $\mathrm{N}$ \\
\hline & & with fault & Y & Y & Y & Y \\
\hline & \multicolumn{2}{|l|}{ Unbalanced loading (at BUS-12) } & Y & Y & $\mathrm{N}$ & $\mathrm{N}$ \\
\hline
\end{tabular}

\section{Conclusions}

WABP systems are essential for maintaining elevated reliability of the protection system under faults and power system contingencies like power swing, load encroachment and unbalanced loading. This paper proposes MPMU based ASWABP scheme, that can detect the transmission line fault, identifies the parent bus, faulty branch \& relays to be supervised and classify the type of fault during power system disturbances. WSCC-9 bus and IEEE-14 bus systems are considered as test systems. Results have shown that the proposed methodology is immune to variation in fault parameters. Also, it can achieve the security-dependability balance during faults and power system contingencies to assist system protection center (SPC) in taking appropriate decision to any catastrophic situation in case of failure in the main protection system. Comparative studies performed have validated that the proposed scheme is superior to the conventional distance protection in terms of enhanced reliability. Futhermore, comparative studies have shown the necessity of the proposed scheme as a supervised wide-area backup system for distance protection to enhance the reliability.

\section{Authors' contributions}

BM has developed and modeled the proposed ASWABP algorithm. PW developed the Fourier analysis for fault classification methodology. SDS has made substantial contributions to simulated IEEE networks. MJBR has been technical adviser for the total work and DKM has supported us in interpreting the simulation results for fault analysis. All authors read and approved the final manuscript.

\section{Competing interests}

The authors declare that they have no competing interests

\section{Author details}

${ }^{1}$ Department of Electrical and Electronics Engineering, National Institute of Technology, Tiruchirappalli, Tamilnadu, India. ${ }^{2}$ Department of Electrical and Electronics Engineering, Birla Institute of Technology, Mesra, Ranchi, India.
Received: 10 March 2017 Accepted: 13 May 2017

Published online: 02 June 2017

\section{References}

1. Report on Grid Disturbance on 30th July 2012 and Grid Disturbance on 31st July 2012, August 2012, India. http://www.cercind.gov.in/2012/orders/Final_ Report_Grid_Disturbance.pdf

2. Final Report on the August 14, 2003 Blackout in the United States and Canada: Causes and Recommendations, Canada, April 2004. http://energy. gov/sites/prod/files/oeprod/DocumentsandMedia/BlackoutFinal-Web.pdf.

3. Gunasegaran, M. K., Tan, C. K., Bakar, A. H. A., Mokhlis, H., \& Illias, H. A. (2015). Progress on power swing blocking schemes and the impact of renewable energy on power swing characteristics: a review. Renewable and Sustainable Energy Reviews, 52, 280-288.

4. Nayak, P. K., Pradhan, A. K., \& Bajpai, P. (2015). Secured zone 3 protection during tressed condition. IEEE Transactions on Power Delivery, 30(1), 89-96.

5. Kundu, P., \& Pradhan, A. K. (2014). Synchrophasor-assisted zone 3 operation. IEEE Transactions on Power Delivery, 29(2), 660-667.

6. Phadke, A. G., \& Thorpe, J. S. (2008). Synchronized phasor measurements and their applications. Springer: power electronics and power systems series (p. 207).

7. Bruno, S., De Benedictis, M., Scala, M. L., Lamonaca, S., Rotondo, G., \& Stecchi, U. (2010). Adaptive relaying to balance protection dependability with power system security (pp. 482-487). Listvyanka: IEEE Region 8th International Conference on Computational Technologies in Electrical and Electronics Engineering (SIBIRCON).

8. Horowitz, S. H., Phadke, A. G., \& Thorpe, J. S. (1988). Adaptive transmission system relaying. IEEE Transactions on Power Delivery, 3(4), 1436-1445.

9. Rockefeller, G. D., Wagner, C. L., Linders, J. R., Hicks, K. L., \& Rizy, D. T. (1988). Adaptive transmission relaying concepts for improved performance. IEEE Transactions on Power Delivery, 3(4), 1446-1458.

10. Kundu, P., \& Pradhan, A. K. (2014). Wide area measurement based protection support during power swing. International Journal of Electrical Power \& Energy Systems, 63, 546-554.

11. Kundu, P., \& Pradhan, A. K. (2015). Online identification of protection element failure using wide area measurements. IET Generation Transmission and Distribution, 9(2), 115-123.

12. Tan, J. C., Crossley, P. A., Kirschen, D., Goody, J., \& Downes, J. A. (2000). An expert system for the backup protection of a transmission network. IEEE Transactions on Power Delivery, 15(2), 508-514.

13. Tong, X., Wang, X., Wang, R., Huang, F., Dong, X., Hopkinson, K. M., \& Song, G. (2013). The study of a regional decentralized peer-to-peer negotiationbased wide-area backup protection multi-agent system. IEEE Transactions on Smart Grid, 4(2), 1197-1206. 
14. Giovanini, R., Hopkinson, K., Coury, D. V., \& Thorp, J. S. (2006). A primary and backup cooperative protection system based on wide area agents. IEEE Transactions on Power Delivery, 21(3), 1222-1230.

15. Zare, J., Aminifar, F., \& Sanaye-Pasand, M. (2015). Synchrophasor-based widearea backup protection scheme with data requirement analysis. IEEE Transactions on Power Delivery, 30(3), 1410-1419.

16. Zare, J., Aminifar, F., \& Sanaye-Pasand, M. (2015). Communication constrained regionalization of power systems for synchrophasor based wide-area backup protection scheme. IEEE Transactions on Smart Grid, 6(3), 1530-1538.

17. Kundu, P., \& Pradhan, A. K. (2015). Wide-area backup protection using weighted apparent impedance (pp. 675-679). Bhubaneswar: IEEE Power, Communication and Information Technology Conference (PCITC).

18. Nayak, P. K., Pradhan, A. K., \& Bajpai, P. (2014). Wide-area measurementbased backup protection for power network with series compensation. IEEE Transactions on Power Delivery, 29(4), 1970-1977.

19. Hinge, T., \& Dambhare, S. S. (2014). Secure backup protection of transmission line using WAMS. IEEE international conference on power electronics, drives and energy systems (PEDES) (pp. 1-6).

20. Hall, I., Beaumont, P. G., Baber, G. P., Shuto, I., Saga, M., Okuno, K., \& Uo, H. (2003). New line current differential relay using GPS synchronization. IEEE Bologna Power Tech Conference Proceedings, 3, 23-26.

21. Li, H. Y., Southern, E. P., Crossley, P. A., Potts, S., Pickering, S. D. A., Caunce, B. R. J., \& Weller, G. C. (1997). A new type of differential feeder protection relay using the global positioning system for data synchronization. IEEE Transactions on Power Delivery, 12(3), 1090-1099.

22. Sheng, S., Li, K. K., Chan, W. L., Xiangjun, Z., \& Xianzhong, D. (2006). Agentbased self-healing protection system. IEEE Transactions on Power Delivery, $21(2), 610-618$.

23. Ma, J., Liu, C., \& Thorp, J. S. (2016). A wide-area backup protection algorithm based on distance protection fitting factor. IEEE Transactions on Power Delivery, 31(5), 2196-2205.

24. Reddy, M. J., \& Mohanta, D. K. (2008). Adaptive-neuro-fuzzy inference system approach for transmission line fault classification and location incorporating effects of power swings. IET Generation, Transmission \& Distribution, 2(2), 235-244.

25. Task force on Harmonics Modeling and Simulation. (1996). Modeling and simulation of the propagation of harmonics in electric power networks: Iconcepts, models, and simulation techniques. IEEE Transactions on Power Delivery, 11(1), 452-465.

26. Task force on Harmonics Modeling and Simulation. (1996). Modeling and simulation of the propagation of harmonics in electric power networks: IIsample systems and examples. IEEE Transactions on Power Delivery, 11(1), 466-474.

27. Mallikarjuna, B., Rounak, M., Diptak, P., Jaya Bharata Reddy, M., \& Mohanta, D. K. (2016). An adaptive secure-dependable wide-area backup protection scheme for transmission lines using multi-phasor measurement units. Trivandrum: IEEE International Conference on Power Electronics, Drives and Energy Systems.

28. Kundur, P. (1994). Power system stability and control. New York: McGraw-Hill: Epri Power System Engineering Series.

29. Neyestanaki, M. K., \& Ranjbar, A. M. (2015). An adaptive PMU-based wide area backup protection scheme for power transmission lines. IEEE Transactions on Smart Grid, 6(3), 1550-1559.

30. Anderson, P. M. (1999). Power System Protection. IEEE Press, New York.

31. Adamiak, M. G., Apostolov, A. P., Begovic, M. M., Henville, C. F., Martin, K. E. Michel, G. L., Phadke, A. G., \& Thorp, J. S. (2006). Wide area protection technology and infrastructures. IEEE Transactions on Power Delivery, $21(2), 601$

32. Zima, M., Krause, T., Andersson, G. (2003). Evaluation of system protection schemes, wide area monitoring and control systems (pp. 754-759). Hong Kong: 6th International Conference on Advances in Power System Control, Operation and Management (APSCOM).

\section{Submit your manuscript to a SpringerOpen ${ }^{\circ}$ journal and benefit from:}

- Convenient online submission

- Rigorous peer review

- Open access: articles freely available online

- High visibility within the field

- Retaining the copyright to your article

Submit your next manuscript at springeropen.com 